WU B 95-09

\title{
VIRTUAL COMPTON SCATTERING OFF PROTONS AT MODERATELY LARGE MOMENTUM TRANSFER
}

\author{
P. Kroll*, M. Schürmann \\ Fachbereich Physik, Universität Wuppertal, \\ Gaußstrasse 20, D-42097 Wuppertal, Germany \\ P. A. M. Guichon ${ }^{\dagger}$ \\ SPhN-DAPNIA, CE Saclay, \\ F-91191 Gif sur Yvette, France
}

(February 1, 2008)

\begin{abstract}
The amplitudes for virtual Compton scattering off protons are calculated within the framework of the diquark model in which protons are viewed as being built up by quarks and diquarks. The latter objects are treated as quasi-elementary constituents of the proton. Virtual Compton scattering, electroproduction of photons and the Bethe-Heitler contamination are discussed for various kinematical situations. We particularly emphasize the rôle of the electron asymmetry for measuring the relative phases between the virtual Compton and the Bethe-Heitler amplitudes. It is also shown that the model is able to describe very well the experimental data for real Compton scattering off protons.
\end{abstract}

*kroll@wpts0.physik.uni-wuppertal.de

†pampam@phnx7.saclay.cea.fr 


\section{INTRODUCTION}

The understanding of the structure of the nucleon is a fundamental task of particle physics. Instead of inclusive reactions where the various nucleon constituents contribute incoherently, one may use exclusive reactions to study the structure of the nucleon. The simplest exclusive quantities are the electromagnetic form factors of the nucleon, which can be measured in elastic electron-proton and/or electron-deuteron scattering. The cross section for unpolarized elastic electron-proton scattering is well known up to a momentum transfer $Q^{2}$ of $33 \mathrm{GeV}^{2}$ [目]. For large momentum transfer $\left(\geq 8.8 \mathrm{GeV}^{2}\right)$ only the magnetic form factor of the proton has been determined because the contribution of the electric one is suppressed in the cross section by $1 / Q^{2}$. In a recent SLAC experiment [2] the electric and magnetic form factors of the proton have been measured separately in the $Q^{2}$ range from $1.75 \mathrm{GeV}^{2}$ to $8.83 \mathrm{GeV}^{2}$. The data on the neutron form factors is very poor at large momentum transfer.

The next simplest process to probe the structure of the nucleon is Compton scattering off protons at large momentum transfers. The cross section for real Compton scattering has been measured up to initial photon energies of $6 \mathrm{GeV}$ [3]. The process $e p \rightarrow e p \gamma$ offers the possibility to study virtual Compton (VC) scattering and its interference with the Bethe-Heitler (BH) process. At present there is no experimental data available. In the near future, however, CEBAF will offer the possibility to measure the process $e p \rightarrow e p \gamma$ for incident electron energies smaller than $6 \mathrm{GeV}$ [ [W]. The investigation of the high energy region requires a new type of electron accelerator [5]. We note that the $\mathrm{BH}$ contribution to the procces $e p \rightarrow e p \gamma$ is well known [6].

The general framework for calculating large momentum transfer processes has been developed by Brodsky and Lepage [7]. Using the QCD factorization theorem for exclusive reactions [7,8], one can write baryon form factors and Compton scattering off baryons as multidimensional integrals over a product of distribution amplitudes (DA) and elementary amplitudes which represent the scattering of constituents in collinear approximation. A DA is the valence Fock-state wave function integrated over transverse momenta $\mathbf{k}_{\perp}^{(i)}$ up to a scale of order $Q^{2}$. It specifies the distribution of the longitudinal momentum fractions the constituents carry inside their parent hadrons. Contributions from higher Fock states are suppressed by powers of $\alpha_{s} / Q^{2}$ in exclusive processes where $\alpha_{s}$ is the strong coupling constant.

Some information about DAs containing the non-perturbative physics, is obtained from QCD sum rules [9 11] and from lattice gauge theory [12,13]. The absolute normalization of the nucleon wave function has been estimated: QCD sum rules provide a value of $f_{N}=(5.0 \pm 0.3) \cdot 10^{-3} \mathrm{GeV}^{2}$ for the wave function at the origin of the configuration space whereas $f_{N}=(2.9 \pm 0.6) \cdot 10^{-3} \mathrm{GeV}^{2}$ has been obtained from lattice gauge theory. Also the moments of the nucleon's DA have been calculated up to order three. These moments are used to constrain the first five coefficients in an expansion of the DA over the eigenfunctions of the evolution equation which are linear combinations of the Appell polynomials. It is obvious that the DAs do not unambiguously follow from the few error-burdened moments provided by QCD sum rules or lattice gauge theories (a careful analysis of this problem has been performed by Bergmann and Stefanis [14]). Therefore, the DAs to be found in the literature, e. g., [10,11,14 are to be considered as models. Anyway, the striking feature of 
these DAs is their strong asymmetry which gives preference to positive helicity $u$ quarks in positive helicity protons. The asymmetry of these DAs is the source of theoretical inconsistencies in applications of the Brodsky-Lepage model, because it strongly enhances the contributions from the end-point regions where $\alpha_{s}$ is large and, hence, the use of perturbation theory is unjustified. Recently it has been shown [15 17] that perturbative calculations become theoretically self-consistent for momentum transfers as low as a few $\mathrm{GeV}^{2}$ when the transverse separations of the constituents as well as a Sudakov factor, comprising gluonic radiative corrections, are taken into account. If one also includes the intrinsic transverse momentum dependence of the hadronic wave function perturbative calculations may become self-consistent even for momentum transfers as low as 1 to $2 \mathrm{GeV}^{2}$ in the case of the pion 18] and at about $7-9 \mathrm{GeV}^{2}$ for the nucleon [17]. On the other hand, the inclusion of the transverse degrees of freedom and of the Sudakov factor leads to substantial suppressions of the perturbative contributions in the few $\mathrm{GeV}$ region which are particularly strong for the asymmetric DAs.

To leading order perturbation theory many processes have been calculated within the standard approach of Brodsky and Lepage [7], among them is the pion's form factor, the magnetic form factor of the nucleon, two-photon annihilation into meson pairs and into proton-antiproton as well as Compton scattering off protons. To next to leading order in $\alpha_{s}$ only the pion's form factor and two photon annihilation into meson pairs have been studied. It was shown [19] that the corrections to the lowest order predictions for $\gamma \gamma \rightarrow M \bar{M}$ become sufficiently small $(\leq 25 \%)$ only for very large center of mass energies $\sqrt{s} \leq 10 \mathrm{GeV}$. Other important reactions like elastic proton-proton scattering seem to be beyond feasibility with present-day techniques of computing very large numbers of Feynman diagrams. There are also unsolved theoretical complications with pinch singularities [8].

There are two characteristic features of the Brodsky-Lepage model, commonly termed the hard scattering approach (HSA): the dimensional counting rules and the conservation of hadronic helicities. The latter feature implies that any helicity flip amplitude is zero and, hence, any single spin asymmetry too. The helicity sum rule is a consequence of utilizing the collinear approximation and of dealing with (almost) massless quarks which conserve their helicities when interacting with gluons. Whereas the dimensional counting rules are in reasonable agreement with experiment, seems the helicity sum rule to be violated by $20-25 \%$. For instance, the polarization parameter $P$ in elastic proton-proton scattering is by no means zero. On the contrary, it is rather large $(20-30 \%)$ at moderately large values of momentum transfer $\left(p_{T}>2 \mathrm{GeV} / \mathrm{c}\right)$ [20]. Even worse, the data show the tendency of further rise of the polarization with increasing $p_{T}$. Such peculiar polarization phenomena have also been observed in many (moderately) large $p_{T}$ inclusive reactions like hyperon production in nucleon-nucleon collisions or $p p \rightarrow \pi^{ \pm} X$ [21]. The prevailing opinion is that also these phenomena cannot be explained in terms of perturbative QCD (see, for example, Ref. [22, 23]), rather they are generated by an interplay of perturbative and non-perturbative physics.

A model which takes into considerations non-perturbative effects, has been proposed by us in a series of papers 24 27]. In that model baryons are viewed as made of quarks and diquarks, the latter being treated as quasi-elementary constituents which partly survive medium hard collisions. The composite nature of the diquarks is taken into account by diquark form factors. Diquarks are an effective description of correlations in the wave 
functions and constitute a particular model for non-perturbative effects. The diquark model has been applied to a variety of processes and successfully confronted to data. Among these applications is a recent study of the nucleon's electromagnetic form factors [27]. In fact, predictions are achieved for both the magnetic and the electric form factors. For the latter quantity no result is obtained in the pure quark hard scattering model because it requires helicity flips of the nucleon and, in so far, the electric form factor or better the Pauli form factor, also represents a polarization effect. In the diquark model helicity flips are generated through spin $1(\mathrm{~V})$ diquarks. The diquark model is designed such that it turns into the theoretically well established pure quark picture asymptotically. In so far the pure quark picture of Brodsky-Lepage and the diquark model do not oppose each other, they are not alternatives but rather complements.

The purpose of this paper is to discuss virtual Compton scattering off protons and its interference with the Bethe-Heitler process where the photon is emitted by the electrons (see Fig. 1). The Compton amplitudes are calculated within the framework of the diquark model with the parameters taken from previous studies [26,27]. Real and virtual Compton scattering are the two simplest experimentally accessible processes in which the integrals over the longitudinally momentum fractions yield imaginary parts. The reason is that there are kinematical regions where internal quarks and gluons can go on mass shell. It was shown in 28 that the lowest order predictions of perturbative QCD for photon induced processes need no resummation of Sudakov corrections. The essential point is that the singularities do not pinch as is the case in baryon-baryon scattering. The appearance of imaginary parts to leading order in $\alpha_{s}$ is a non-trivial prediction of perturbative QCD. The process $e p \rightarrow e p \gamma$ with polarized incoming electrons offers the possibility to determine the phases of the Compton amplitudes as a function of the center of mass scattering angle, by investigating the interference of the virtual Compton with the Bethe-Heitler process. In principle, the measurements of other spin observables through the use of polarized targets and polarized beams would offer further possibilities of crucially testing the applicability of the HSA in the few GeV region. The process $e p \rightarrow e p \gamma$ has been studied in the framework of the pure quark HSA by Farrar and Zhang [29]. Real Compton scattering has, on the other side been investigated by two groups [29,30]. Unfortunately, the results obtained by the two groups deviate from each other substantially. It is suspected that the discrepancies are caused by an improper treatment of propagator singularities in [29]. The kinematical flexibility of $e p \rightarrow e p \gamma$ allows to test the predictions with respect to the virtual photon mass, to the center of mass energy and to the momentum transfer.

In Sect. II a detailed discussion of the kinematics and the various observables of the reaction $e p \rightarrow e p \gamma$ is given. A short description of the diquark model for exclusive reactions is presented in Sect. III followed by a brief discussion of the results for real Compton scattering (Sect. [V]). The predictions for the cross section of VC scattering and for the unpolarized cross section of the photon electroproduction $e p \rightarrow e p \gamma$ are discussed in Sect. $\mathrm{V}$. This section also includes a discussion of the interference between the $\mathrm{VC}$ amplitudes and the $\mathrm{BH}$ ones. The case of polarized electron beams and its physics implications is investigated in Sect. 6 . The paper terminates with a few concluding remarks (Sect. 7). 


\section{KINEMATICS OF ELECTROPRODUCTION OF PHOTONS}

The process $e p \rightarrow e p \gamma$ receives contributions from the $\mathrm{VC}$ and from the $\mathrm{BH}$ processes. The kinematics and the helicities are specified in Fig. 1. We work in the center of mass $(\mathrm{CM})$ frame of the final state photon and proton. Neglecting the electron mass the different particle momenta can be chosen to be

$$
\begin{aligned}
& k^{\mu}=\left(k_{0}, k_{0} \sin \alpha \cos \phi, k_{0} \sin \alpha \sin \phi, k_{0} \cos \alpha\right) \\
& k^{\prime \mu}=\left(k_{0}-q_{0}, k_{0} \sin \alpha \cos \phi, k_{0} \sin \alpha \sin \phi, k_{0} \cos \alpha-|\mathbf{p}|\right) \\
& q^{\mu}=\left(q_{0}, 0,0,|\mathbf{p}|\right) \\
& q^{\prime \mu}=q_{0}^{\prime}(1, \sin \theta, 0, \cos \theta) \\
& p^{\mu}=\left(p_{0}, 0,0,-|\mathbf{p}|\right) \\
& p^{\prime \mu}=\left(p_{0}^{\prime},-q_{0}^{\prime} \sin \theta, 0,-q_{0}^{\prime} \cos \theta\right)
\end{aligned}
$$

in that frame. $\phi$ is the angle between the hadronic and leptonic scattering plane, and $\theta$ is the scattering angle of the outgoing real photon. The polarization vector $\epsilon_{f}$ of the outgoing photon is given by

$$
\epsilon_{f}\left(\mu^{\prime}\right)=\frac{1}{\sqrt{2}}\left(0,-\mu^{\prime} \cos \theta,-i, \mu^{\prime} \sin \theta\right) .
$$

$q_{0}, p_{0}, q_{0}^{\prime}, p_{0}^{\prime}$ and $|\mathbf{p}|$ are related to the Mandelstam variable $s=(p+q)^{2}$ and to the virtuality $\left(q^{2}=-Q^{2}\right)$ of the exchanged photon by

$$
\begin{array}{rr}
q_{0}=\frac{s-Q^{2}-m^{2}}{2 \sqrt{s}} & q_{0}^{\prime}=\frac{s-m^{2}}{2 \sqrt{s}} \\
p_{0}=\frac{s+Q^{2}+m^{2}}{2 \sqrt{s}} & p_{0}^{\prime}=\frac{s+m^{2}}{2 \sqrt{s}} \\
|\mathbf{p}|=\frac{1}{2 \sqrt{s}} \Lambda\left(s,-Q^{2}, m^{2}\right)
\end{array}
$$

where $m$ is the nucleon mass. The Mandelstam function $\Lambda$ is defined by

$$
\Lambda(x, y, z)=\sqrt{x^{2}+y^{2}+z^{2}-2 x y-2 x z-2 y z} .
$$

Momentum conservation leads to the following relations

$$
k_{0} \sin \alpha=\frac{Q}{2|\mathbf{p}|} \sqrt{\left(2 k_{0}-q_{0}\right)^{2}-|\mathbf{p}|^{2}}, \quad k_{0} \cos \alpha=\frac{1}{2|\mathbf{p}|}\left(q_{0}\left(2 k_{0}-q_{0}\right)+|\mathbf{p}|^{2}\right) .
$$

The other frequently used reference frame is the laboratory frame defined by $\mathbf{p}=0$. In that frame one has the following useful relations:

$$
\begin{aligned}
& s=-Q^{2}+m^{2}+2 m k_{0 L} \\
& Q^{2}=4 k_{0 L} k_{0 L}^{\prime} \sin ^{2} \frac{\theta_{e}^{L}}{2}
\end{aligned}
$$


where $k_{0 L}$ and $k_{0 L}^{\prime}$ are the energies of the inital and final state electrons in the LAB and $\theta_{e}^{L}$ is the electron scattering angle. ¿From the theoretical point of view the most significant variables are the invariants $s, t, Q^{2}$ and the polarization of the virtual photon in the VC process

$$
\varepsilon:=\frac{\left(q_{0}-2 k_{0}\right)^{2}-|\mathbf{p}|^{2}}{\left(q_{0}-2 k_{0}\right)^{2}+|\mathbf{p}|^{2}}=\left[1+2 \frac{\left(k_{0 L}-k_{0 L}^{\prime}\right)^{2}+Q^{2}}{Q^{2}} \tan ^{2} \frac{\theta_{e}^{L}}{2}\right]^{-1} .
$$

The unpolarized differential cross section for the reaction $e p \rightarrow e p \gamma$ can be written as

$$
\frac{d^{4} \sigma}{d s d Q^{2} d \phi d t}(e p \rightarrow e p \gamma)=\frac{1}{128(2 \pi)^{4} k_{0 L}^{2} m^{2} \Lambda\left(s,-Q^{2}, m^{2}\right)} \frac{1}{4} \sum_{\substack{\nu \nu^{\prime} \\ \mu^{\prime} \lambda^{\prime} \lambda}}\left|T_{\nu^{\prime} \mu^{\prime} \lambda^{\prime}, \nu \lambda}^{B H}+T_{\nu^{\prime} \mu^{\prime} \lambda^{\prime}, \nu \lambda}^{V C}\right|^{2}
$$

where $T^{B H}$ and $T^{V C}$ denote the helicity amplitudes for the $\mathrm{BH}$ and for the VC contributions to the electroproduction of photons, respectively. In the one-photon approximation the latter read (see Fig. 1)

$$
T_{\nu^{\prime} \mu^{\prime} \lambda^{\prime}, \nu \lambda}^{V C}=\frac{\sqrt{4 \pi \alpha}}{Q^{2}} \delta_{\nu^{\prime} \nu} \bar{u}\left(k^{\prime}, \nu^{\prime}\right) \gamma^{\alpha} u(k, \nu)\left\langle q^{\prime} \mu^{\prime}, p^{\prime} \lambda^{\prime}\left|j_{\alpha}^{V C}\right| p \lambda\right\rangle .
$$

In the CM frame the two-body $s$-channel helicity amplitudes $\Phi_{i}:=M_{\mu^{\prime} \lambda^{\prime}, \mu \lambda}\left(s, t, Q^{2}\right)$ for $\mathrm{VC}$ scattering are defined by

$$
M_{\mu^{\prime} \lambda^{\prime}, \mu \lambda}=\epsilon^{\alpha}(\mu)\left\langle q^{\prime} \mu^{\prime}, p^{\prime} \lambda^{\prime}\left|j_{\alpha}^{V C}\right| p \lambda\right\rangle
$$

where $\epsilon_{i}^{\alpha}$ is the polarization vector of the virtual photon

$$
\epsilon_{i}^{\alpha}(0)=\frac{1}{Q}\left(|\mathbf{p}|, 0,0, q_{0}\right) \quad \epsilon_{i}^{\alpha}( \pm 1)=\mp \frac{1}{\sqrt{2}}(0,1, i, 0) .
$$

Current conservation leads to a relation between the 0-and 3-components of the hadronic current matrix element. The projection of the electron current on the polarization vectors is straightforward and leads to the following relation between the $\mathrm{VC}$ contribution to the photon electroproduction amplitudes and the VC helicity amplitudes

$$
\begin{array}{r}
T_{\nu^{\prime} \mu^{\prime} \lambda^{\prime}, \nu \lambda}^{V C}=\frac{1}{Q} \sqrt{\frac{4 \pi \alpha}{1-\varepsilon}}\left\{\frac{1}{\sqrt{2}}[\sqrt{1+\varepsilon}+2 \nu \sqrt{1-\varepsilon}] e^{-i \phi} M_{\mu^{\prime} \lambda^{\prime},+1 \lambda}-\sqrt{2 \varepsilon} M_{\mu^{\prime} \lambda^{\prime}, 0 \lambda}\right. \\
\left.\left.-\frac{1}{\sqrt{2}}[\sqrt{1+\varepsilon}-2 \nu \sqrt{1-\varepsilon}] e^{i \phi} M_{\mu^{\prime} \lambda^{\prime},-1 \lambda}\right)\right\} \delta_{\nu^{\prime} \nu} .
\end{array}
$$

Due to parity invariance there are only 12 independent $s$-channel helicity amplitudes contributing to the process $\gamma^{*} p \rightarrow \gamma p$. These are conveniently denoted by $\Phi_{i}, i=1,12$ :

$$
\begin{aligned}
& \Phi_{1}=M_{+1+\frac{1}{2}, 1+\frac{1}{2}} \quad \Phi_{5}=M_{+1-\frac{1}{2}, 1-\frac{1}{2}} \quad \Phi_{9}=M_{+1+\frac{1}{2}, 0+\frac{1}{2}} \\
& \Phi_{2}=M_{-1-\frac{1}{2}, 1+\frac{1}{2}} \quad \Phi_{6}=M_{-1+\frac{1}{2}, 1-\frac{1}{2}} \quad \Phi_{10}=M_{-1-\frac{1}{2}, 0+\frac{1}{2}} \\
& \Phi_{3}=M_{-1+\frac{1}{2}, 1+\frac{1}{2}} \quad \Phi_{7}=M_{-1-\frac{1}{2}, 1-\frac{1}{2}} \quad \Phi_{11}=M_{-1+\frac{1}{2}, 0+\frac{1}{2}} \\
& \Phi_{4}=M_{+1-\frac{1}{2}, 1+\frac{1}{2}} \quad \Phi_{8}=M_{+1+\frac{1}{2}, 1-\frac{1}{2}} \quad \Phi_{12}=M_{+1-\frac{1}{2}, 0+\frac{1}{2}}
\end{aligned}
$$


$\Phi_{1}-\Phi_{8}$ correspond to transverse polarization of the virtual photon and $\Phi_{9}-\Phi_{12}$ to longitudinally polarized photons. In the real photon limit the latter four amplitudes vanish. In that limit time reversal invariance reduces the number of independent amplitudes even further: $\left(\Phi_{7}=\Phi_{3}, \Phi_{8}=-\Phi_{4}\right)$. The VC contribution to the $e p \rightarrow e p \gamma$ cross-section can be decomposed as follows

$$
\begin{aligned}
& \left.\frac{d^{4} \sigma}{d s d Q^{2} d \phi d t}\right|_{V C}=\frac{\alpha\left(s-m^{2}\right)}{4(2 \pi)^{2} k_{0 L}^{2} m^{2} Q^{2}(1-\varepsilon)} \\
& \times\left(\frac{d \sigma_{T}}{d t}+\varepsilon \frac{d \sigma_{L}}{d t}+\varepsilon \cos 2 \phi \frac{d \sigma_{T T}}{d t}+\sqrt{2 \varepsilon(1+\varepsilon)} \cos \phi \frac{d \sigma_{L T}}{d t}\right)
\end{aligned}
$$

where the partial cross-sections read:

i) The cross-section for transverse photons (which, at $Q^{2}=0$, reduces to the unpolarized cross section $d \sigma / d t$ for real Compton scattering)

$$
\frac{d \sigma_{T}}{d t}=\frac{c}{2} \sum_{i=1}^{8}\left|\Phi_{i}\right|^{2} .
$$

ii) The cross-section for longitudinal photons

$$
\frac{d \sigma_{L}}{d t}=c \sum_{i=9}^{12}\left|\Phi_{i}\right|^{2}
$$

iii) The transverse-transverse interference term

$$
\frac{d \sigma_{T T}}{d t}=-\frac{c}{2} \Re e\left[\Phi_{1}^{*} \Phi_{7}-\Phi_{2}^{*} \Phi_{8}+\Phi_{3}^{*} \Phi_{5}-\Phi_{4}^{*} \Phi_{6}\right]
$$

iv) The longitudinal-transverse interference term

$$
\frac{d \sigma_{L T}}{d t}=-\frac{c}{\sqrt{2}} \Re e\left[\Phi_{9}^{*}\left(\Phi_{1}-\Phi_{7}\right)+\Phi_{10}^{*}\left(\Phi_{2}+\Phi_{8}\right)+\Phi_{11}^{*}\left(\Phi_{3}-\Phi_{5}\right)+\Phi_{12}^{*}\left(\Phi_{4}+\Phi_{6}\right)\right] .
$$

The phase space factor $c$ is given by

$$
c=\frac{1}{16 \pi\left(s-m^{2}\right) \Lambda\left(s,-Q^{2}, m^{2}\right)}
$$

The Bethe-Heitler amplitudes read (see Fig. 1)

$$
T_{\nu^{\prime} \mu^{\prime} \lambda^{\prime}, \nu \lambda}^{B H}=\frac{4 \pi \alpha}{t} L_{\alpha}^{B H}\left\langle p^{\prime} \lambda^{\prime}\left|j^{\alpha}\right| p \lambda\right\rangle
$$

where the $\gamma^{*} p \rightarrow p$ current matrix element is expressed in terms of the magnetic form factor of the proton $G_{M}$ and the Pauli form factor $F_{2}$ by:

$$
\left\langle p^{\prime} \lambda^{\prime}\left|j^{\alpha}\right| p \lambda\right\rangle=\sqrt{4 \pi \alpha} \bar{u}\left(p^{\prime}, \lambda^{\prime}\right)\left(\gamma^{\alpha} G_{M}(t)-\frac{\kappa_{p}}{2 m}\left(p^{\prime}+p\right)^{\alpha} F_{2}(t)\right) u(p, \lambda) .
$$


The electric form factor $G_{E}$ is related to $F_{2}$ by $G_{E}=G_{M}-\kappa_{p}(1+\tau) F_{2}$ where $\tau=-t / 4 m^{2}$. The leptonic current $L_{\alpha}^{B H}$ is given by

$$
\begin{aligned}
L_{\alpha}^{B H}=\left\{2 \frac{k^{\prime} \cdot \epsilon_{f}^{*}}{s_{k^{\prime} q^{\prime}}}+2 \frac{k \cdot \epsilon_{f}^{*}}{t_{k q^{\prime}}}\right\} \bar{u}\left(k^{\prime}, \nu^{\prime}\right) \gamma^{\alpha} u(k, \nu) & \\
& +\bar{u}\left(k^{\prime}, \nu^{\prime}\right)\left\{\frac{\phi_{f}^{*} \phi^{\prime} \gamma_{\alpha}}{s_{k^{\prime} q^{\prime}}}-\frac{\gamma_{\alpha} \phi^{\prime} \phi_{f}^{*}}{t_{k q^{\prime}}}\right\} u(k, \nu)
\end{aligned}
$$

where $s_{a b}=(a+b)^{2}$ and $t_{a b}=(a-b)^{2}$. In the soft photon approximation $q^{\prime} \rightarrow 0$, the first term in (2.22) is the usual Bremsstrahlung contribution. Putting all together the $\mathrm{BH}$ contribution to the process $e p \rightarrow e p \gamma$ may be written in a form similar to that of the Rosenbluth cross section for elastic ep scattering:

$$
\begin{aligned}
& \left.\frac{d^{4} \sigma}{d s d Q^{2} d \phi d t}\right|_{B H}=\frac{\alpha^{3}}{4 \pi k_{0 L}^{2} m^{2} \Lambda\left(s,-Q^{2}, m^{2}\right) t^{2}} \\
& \times\left\{A\left(s_{k p}, s, Q^{2}, \phi, t\right) \frac{G_{E}^{2}(t)+\tau G_{M}^{2}(t)}{1+\tau}+B\left(s_{k p}, s, Q^{2}, \phi, t\right) G_{M}^{2}(t)\right\} .
\end{aligned}
$$

The functions $A$ and $B$ are given by

$$
\begin{aligned}
& A\left(s_{k p}, s, Q^{2}, \phi, t\right)=m^{2}\left(\frac{s_{k^{\prime} q^{\prime}}}{t_{k q^{\prime}}}+\frac{t_{k q^{\prime}}}{s_{k^{\prime} q^{\prime}}}\right)+\frac{t}{s_{k^{\prime} q^{\prime}}}\left(s_{k p}-s-Q^{2}-2 m^{2}\right) \\
& -\frac{t}{t_{k q^{\prime}}}\left(s_{k p}+m^{2}\right)+\frac{t}{s_{k^{\prime} q^{\prime}} t_{k q^{\prime}}}\left(2\left(s_{k p}-m^{2}\right)\left(s_{k p}-s-Q^{2}\right)-u\left(s+Q^{2}\right)+m^{2}\left(t+m^{2}\right)\right) \\
& B\left(s_{k p}, s, Q^{2}, \phi, t\right)=\frac{t}{2}\left(\frac{s_{k^{\prime} q^{\prime}}}{t_{k q^{\prime}}}+\frac{t_{k q^{\prime}}}{s_{k^{\prime} q^{\prime}}}\right)-\frac{t^{2} Q^{2}}{s_{k^{\prime} q^{\prime}} t_{k q^{\prime}}}
\end{aligned}
$$

For large $t$ the contribution from the electric form factor is suppressed by the factor $\tau$ as compared to that from the magnetic one. The interference term between the $\mathrm{BH}$ and the VC contribution is a very lengthy expression and we refrain from showing it.

Similar expressions as (2.14) and (2.23) hold also for polarized cross sections. However, most likely only the polarization of the electron beams can be used at high energies. Therefore, we refain from discussing polarized cross sections in this article with the only exceptions of the electron asymmetry $A_{L}$ obtained by reversing the helicity of the beam electrons, and the corresponding proton asymmetry. The discussion of these asymmetries is postponed to Sect. 6 .

\section{THE DIQUARK MODEL}

In the hard-scattering model of Brodsky-Lepage [7] the process $\gamma^{*} p \rightarrow \gamma p$ is expressed by a convolution of DAs with hard-scattering amplitudes calculated in collinear approximation within perturbative QCD. In a collinear situation in which intrinsic transverse momenta are neglected and all constituents of a hadron have momenta parallel to each other and parallel to the momentum of the parent hadron, one may write the valence Fock state of the proton in a covariant fashion (omitting colour indices for convenience) 


$$
|p, \lambda\rangle=f_{S} \varphi_{S}\left(x_{1}\right) B_{S} u(p, \lambda)+f_{V} \varphi_{V}\left(x_{1}\right) B_{V}\left(\gamma^{\alpha}+p^{\alpha} / m\right) \gamma_{5} u(p, \lambda) / \sqrt{3}
$$

$u$ is the spinor of a proton with momentum $p$ and helicity $\lambda$. The two terms in (3.1) represent configurations consisting of a quark and either a spin-isospin zero $(S)$ or a spin-isospin one $(V)$ diquark, respectively. The couplings of the diquarks with the quarks in a proton lead to the flavour functions

$$
B_{S}=u S_{[u, d]} \quad B_{V}=\left[u V_{\{u, d\}}-\sqrt{2} d V_{\{u, u\}}\right] / \sqrt{3} .
$$

The use of covariant spin wave functions has many technical advantages. For instance the calculation of a large set of so-called elementary amplitudes is avoided, one immediately projects onto hadronic states. Another advantage is that only hadronic quantities (spinors, polarization vectors, and so on) appear. The $\mathrm{DA} \varphi_{S(V)}\left(x_{1}\right)$, where $x_{1}$ is the momentum fraction carried by the quark, represents the light-cone wave function integrated over transverse momentum and is defined in such way that

$$
\int_{0}^{1} d x_{1} \varphi_{S,(V)}\left(x_{1}\right)=1 .
$$

The constant $f_{S(V)}$ acts as the value of the configuration space wave function at the origin. Representative Feynman graphs contributing to the hard-scattering amplitudes for the process of interest in this article, are displayed in Fig. 2. The blobs appearing at the $g D, \gamma g D$ and $\gamma \gamma D$ vertices symbolize three-, four- and five-point functions. These n-point functions are evaluated for point-like diquarks and the results are multiplied with phenomenological vertex functions (diquark form factors) which take into account the composite nature of the diquarks. Admittedly, that recipe is a rather crude approximation for $n \geq 4$. Since the contributions from the n-point functions for $n \geq 4$ only represent small corrections to the final results that recipe is perhaps sufficiently accurate. The perturbative part of the model, i.e. the coupling of gluons (and photons) to diquarks follows standard prescriptions, see e. g., [31]. The diquark-gluon vertices read (refer to [25,26] for notations)

$$
\begin{aligned}
& \mathrm{SgS}: i g_{s} t_{i j}^{a}\left(p_{1}+p_{2}\right)_{\mu} \\
& \mathrm{VgV}:-i g_{s} t_{i j}^{a}\left\{g_{\alpha \beta}\left(p_{1}+p_{2}\right)_{\mu}-g_{\mu \alpha}\left[(1+\kappa) p_{1}-\kappa p_{2}\right]_{\beta}-g_{\mu \beta}\left[(1+\kappa) p_{2}-\kappa p_{1}\right]_{\alpha}\right\}
\end{aligned}
$$

where $g_{s}=\sqrt{4 \pi \alpha_{s}}$ is the QCD coupling constant. $\kappa$ is the anomalous magnetic moment of the vector diquark and $t^{a}=\lambda^{a} / 2$ the Gell-Mann colour matrix. For the coupling of photons to diquarks one has to replace $g_{s} t^{a}$ by $-\sqrt{4 \pi \alpha} e_{D}$ where $\alpha$ is the fine structure constant and $e_{D}$ is the electrical charge of the diquark in units of the elementary charge. The couplings $D g D$ are supplemented by appropriate contact terms required by gauge invariance

$$
\begin{aligned}
& \gamma \mathrm{SgS}:-2 i e_{0} e_{S} g_{s} t_{i j}^{a} g_{\mu \nu} \\
& \gamma \mathrm{VgV}: i e_{0} e_{V} g_{s} t_{i j}^{a}\left(2 g_{\mu \nu} g_{\alpha \beta}-g_{\mu \beta} g_{\alpha \nu}-g_{\mu \alpha} g_{\beta \nu}\right)
\end{aligned}
$$

As we already mentioned the composite nature of the diquarks is taken into account by phenomenological vertex functions. Advice for the parameterization of the 3-point functions, ordinary diquark form factors, is obtained from the requirement that asymptotically 
the diquark model evolves into the hard scattering model of Brodsky-Lepage. This requirement fixes the asymptotic behaviour of the form factors. Interpolating smoothly between that behaviour and the conventional value of 1 at $Q^{2}=0$, the form factors are actually parametrized as

$$
F_{S}^{(3)}\left(Q^{2}\right)=\frac{Q_{S}^{2}}{Q_{S}^{2}+Q^{2}}, \quad F_{V}^{(3)}\left(Q^{2}\right)=\left(\frac{Q_{V}^{2}}{Q_{V}^{2}+Q^{2}}\right)^{2} .
$$

The asymptotic behaviour of the diquark form factors and the connection to the hard scattering model is discussed in more detail in Ref. [25].

In accordance with the required asymptotic behaviour the $n$-point functions for $n \geq 4$ are parametrized as

$$
F_{S}^{(n)}\left(Q^{2}\right)=a_{S} F_{S}^{(3)}\left(Q^{2}\right), \quad F_{V}^{(n)}\left(Q^{2}\right)=\left(a_{V} \frac{Q_{V}^{2}}{Q_{V}^{2}+Q^{2}}\right)^{n-3} F_{V}^{(3)}\left(Q^{2}\right) .
$$

The constants $a_{S, V}$ are strength parameters. Indeed, since the diquarks in intermediate states are rather far off-shell one has to consider the possibility of diquark excitation and break-up. Both these possibilities would likely lead to inelastic reactions. Therefore, we have not to consider these possibilities explicitly in our approach but excitation and break-up lead to a certain amount of absorption which is taken into account by the strength parameters. Actually, for our numerical studies we use [26,27]

$$
\begin{aligned}
& \varphi_{S}\left(x_{1}\right)=N_{S} x_{1} x_{2}^{3} \exp \left[-b^{2}\left(m_{q}^{2} / x_{1}+m_{S}^{2} / x_{2}\right)\right] \\
& \varphi_{V}\left(x_{1}\right)=N_{V} x_{1} x_{2}^{3}\left(1+5.8 x_{1}-12.5 x_{1}^{2}\right) \exp \left[-b^{2}\left(m_{q}^{2} / x_{1}+m_{V}^{2} / x_{2}\right)\right]
\end{aligned}
$$

and the set of parameters

$$
\begin{aligned}
& f_{S}=73.85 \mathrm{MeV}, Q_{S}^{2}=3.22 \mathrm{GeV}^{2}, \quad a_{S}=0.15 \\
& f_{V}=127.7 \mathrm{MeV}, Q_{V}^{2}=1.50 \mathrm{GeV}^{2}, \quad a_{V}=0.05, \quad \kappa=1.39 ;
\end{aligned}
$$

$\alpha_{s}=12 \pi / 25 \log \left(Q^{2} / \Lambda_{Q C D}\right)$ is evaluated with $\Lambda_{Q C D}=200 \mathrm{MeV}$ and restricted to be smaller than 0.5. The parameters $Q_{S}$ and $Q_{V}$, controlling the size of the diquarks, are in agreement with the higher-twist effects observed in the structure functions of deep inelastic leptonhadron scattering [32] if these effects are modeled as lepton-diquark elastic scattering. The DAs are a kind of harmonic oscillator wave function transformed to the light cone. The masses in the exponentials are constituent masses since they enter through a rest frame wave function. For the quarks we take $330 \mathrm{MeV}$ whereas for the diquarks a value of $580 \mathrm{MeV}$ is used. The oscillator parameter $b$ is taken to be $0.498 \mathrm{GeV}^{-1}$. The constant $\left(N_{S}=\right.$ 25.97; $N_{V}=22.29$ ) are fixed by the requirement (3.3). The more complicated form of the DA $\varphi_{V}$ causes a smaller mean value of $x_{1}$ than obtained for the DA $\varphi_{S}$. The exponentials in the DAs provide strong suppressions in the end-point regions.

According to the above rules the hard scattering amplitudes for the process $\gamma^{*} p \rightarrow \gamma p$ are calculated from the set of lowest order graphs of which a few representatives are shown in Fig. 2. In fact, one has to evaluate 32 graphs for each of the diquarks, $\mathrm{S}$ and V. For comparison, in the pure quark model 366 graphs contribute. The calculation of the 64 
graphs has been carried out using the SUN version of FORM [33]. We have checked that our amplitudes are gauge invariant with respect to the photons and the gluon. The $\gamma^{*} p \rightarrow \gamma p$ helicity amplitudes, decomposed in terms of the various n-point contributions, read

$$
\begin{gathered}
\Phi_{i}=\frac{(4 \pi)^{2} \alpha}{9} C_{F} \int_{0}^{1} d y_{1} \int_{0}^{1} d x_{1}\left\{f_{S}^{2} \varphi_{S}\left(y_{1}\right)\left[4 \Phi_{i}^{(S, 3)}+2 \Phi_{i}^{(S, 4)}+\Phi_{i}^{(S, 5)}\right] \varphi_{S}\left(x_{1}\right)\right. \\
\left.+f_{V}^{2} \varphi_{V}\left(y_{1}\right)\left[2 \Phi_{i}^{(V, 3)}-2 \Phi_{i}^{(V, 4)}+11 \Phi_{i}^{(V, 5)}\right] \varphi_{V}\left(x_{1}\right)\right\}
\end{gathered}
$$

The factor $C_{F}(=4 / 3)$ is the Casimir operator of the fundamental representation of $\mathrm{SU}(3)_{c}$. The n-point contributions generated by the scalar diquarks are explicitely given in the Appendix A. The vector diquark contributions form very lengthy expressions and we refrain form quoting them here; they can be obtained from the authors on request.

\section{RESULTS FOR REAL COMPTON SCATTERING}

The results of the diquark model for real Compton scattering obtained from the DAs and the parameters in (3.8) and (3.9) are shown in Fig. 3 for three different initial photon energies. Note that in the very forward and in the very backward regions the transverse momentum of the outgoing photon is small and, hence, our model which is based on perturbation theory, is not applicable. As compared to the results presented by Kroll, Schürmann and Schweiger 25] there are small modifications of minor importance due to the use of covariant spin wave functions (3.1) and to small changes of the parameters. For the vector diquark contributions the covariant spin wave functions leads to correction terms related to helicity flips of the quarks. Such terms have been neglected in previous work [25].

Although experimental data are available only at energies which are at the limits of applicability of a model based on perturbative QCD, the diquark model is seen to work surprisingly well. The results obtained within the pure quark HSA by Kronfeld and Nižić [30] are of similar quality. It is interesting to see that our predictions for the Compton cross section do not behave as $\sim s^{-6}$ at fixed angles and finite energies as the pure quark HSA predicts. The reason for the deviations from the scaling law is obvious: the various contributions to the cross section exhibit different energy dependences due to the diquark form factors. The diquark model also predicts interesting photon asymmetries and spin correlation parameters (see the discussion in [25]). Even a transverse polarization of the proton, of the order of $10 \%$, is predicted [25]. This comes about as a consequence of the perturbative phases of the amplitudes produced by the propagator poles and of non-zero helicity flip amplitudes generated by the vector diquarks. In the pure quark perturbative approach transverse asymmetries are zero (for massless quarks).

\section{CROSS SECTIONS FOR VC SCATTERING AND FOR ELECTROPRODUCTION OF PHOTONS}

In this section we present the predictions from the diquark model for VC scattering. We select two CM energies, namely $s=5 \mathrm{GeV}^{2}$ which is the high energy end of the CEBAF accelerator (and is at the same time about the lower limit of the applicability of the diquark 
model) and a rather large energy of $s=10 \mathrm{GeV}^{2}$ which may become accessible with future accelerators like, for instance, ELFE.

In Fig. 母 we show the $\gamma^{*} p \rightarrow \gamma p$ cross sections for several values of $Q^{2} / s$. The transverse cross section (2.15) for VC scattering is scaled by $s^{6}$ according to dimensional counting. The most noticeable fact seen in the transverse cross section is the strong decrease with $Q^{2}$, starting from $Q^{2}=0$, the real Compton scattering case. The effect is particular dramatic in the backward direction. It can also be observed from Fig. 田 that, as for real Compton scattering, the dimensional counting rule only holds approximately within a factor of 2 between 5 and $10 \mathrm{GeV}^{2}$. This is a characteristic feature of the diquark model above-mentioned: the diquark form factors lead to a transition from a behaviour $\sim s^{-4}$ (diquark form factors equal unity) to the dimensional counting rule behaviour $\sim s^{-6}$ which is the asymptotic result. The other three cross sections, $\sigma_{L}, \sigma_{T T}$ and $\sigma_{L T}$, are much smaller than the transverse cross section in particular around $90^{\circ}$. Only in the very forward and backward directions these cross sections become sizeable but again these regions, say $|\cos \theta| \geq 0.7$, are outside the hard scattering domain and the application of a model relying on perturbative QCD is suspect. In Fig. 5 the $Q^{2}$ dependence of the $\mathrm{VC}$ cross sections are shown in a hard scattering situation $\left(s=10 \mathrm{GeV}^{2}, \cos \theta=-0.6\right)$ which is accessible at CEBAF. It can be seen that the transition from real Compton scattering to large $Q^{2} \mathrm{VC}$ scattering is non-trivial; a rich structure is predicted. A very interesting phenomenon is the change of the slope of the transverse cross section.

The full VC scattering contribution to the cross section for the electroproduction of

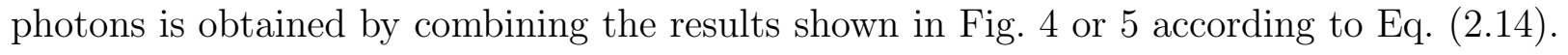

Next we want to investigate the effect of adding coherently the $\mathrm{BH}$ amplitudes to the VC ones. To that purpose we plot in Fig. 6 the difference between the full $e p \rightarrow e p \gamma$ cross section and the VC scattering contribution to it divided by the full cross section. The question of interest is where are the kinematical regions of small $\mathrm{BH}$ contaminations allowing to measure the VC process? As can be seen from Fig. 6 dominance of the VC contributions requires high energies, small values of $|\cos \theta|$ (the actual values depend strongly on $Q^{2}$ and the beam energy $\left.k_{0 L}\right)$ and an out-of-plane experiment, i. e., a large azimuthal angle $\left(\phi \geq 50^{\circ}\right)$. For the actual CEBAF energy of $6 \mathrm{GeV}$ the $\mathrm{VC}$ contribution only dominates in the very backward region and for very small values of $Q^{2}$. Outside the regions of $\mathrm{VC}$ dominance we expect, according to the diquark model, strong BH contaminations (see Fig. 6). We have however not observed pronounced interference phenomena between the $\mathrm{VC}$ and the $\mathrm{BH}$ contributions like the Coulomb-hadronic interference pattern seen in elastic two-body reactions sometimes.

\section{THE ELECTRON ASYMMETRY}

The regions of strong $\mathrm{BH}$ contaminations offer an interesting possibility to measure the phases of the $\mathrm{VC}$ amplitudes relative to those of the $\mathrm{BH}$ amplitudes. As we explain in the Appendix some of the internal quarks, diquarks and gluons may go on mass shell. While these propagator poles are integrable they lead to phases of the VC amplitudes. Real as well as virtual Compton scattering are the simplest reactions in which, to leading order in $\alpha_{S}$, such phases appear [28] and are calculable perturbatively. Thus, it seems to us, that measuring these phases is a very interesting check of the hard scattering approach (with and 
without diquarks).

As a consequence of the phases of the $\mathrm{VC}$ amplitudes, the $\mathrm{VC}$ contributions to the helicity amplitudes of the electroproduction of photons also obtain non-trivial phases beyond the phases due to the azimuthal angle dependence (see Eq. (2.12)). In other words, the perturbative phases manifest themselves in the fact that the $T$ matrix is not self-adjoint. For the $\mathrm{BH}$ process on the other hand, $T=T^{\dagger}$ obviously holds. As we are going to demonstrate information on the absorptive part $T-T^{\dagger}$ can be obtained from the electron asymmetry

$$
A_{L}=\frac{\sigma(+)-\sigma(-)}{\sigma(+)+\sigma(-)}
$$

where $\sigma( \pm)$ are the (differential) cross section for electroproduction of photons with specific helicity of the incoming electron. Since at CEBAF the electron beam is polarized a measurement of the asymmetry seems feasible to us. We emphasize that this possibility of measuring the absorptive part of the $T$ matrix by the electron asymmetry does not depend on our model but is quite general and follows from parity and time reversal invariance merely. As is well-known a one-particle helicity state transforms under the combined parity and time reversal operation as

$$
|\mathbf{k}, \lambda\rangle \longrightarrow \eta(\lambda)|\mathbf{k},-\lambda\rangle
$$

where $\eta(\lambda)$ is \pm 1 depending on $\lambda$, the spin of the particle and on its internal parity. So the combined parity and time reversal operation transform a given helicity state into a state with the same momentum but with reversed helicity. If the interaction is invariant under the parity and time reversal operation the $T$-matrix elements for, say, a $2 \rightarrow 3$ body process satisfy the relation

$$
\begin{aligned}
\left\langle\mathbf{k}_{1}^{\prime}, \lambda_{1}^{\prime} ; \mathbf{k}_{2}^{\prime}, \lambda_{2}^{\prime} ; \mathbf{k}_{3}^{\prime}, \lambda_{3}^{\prime}|T| \mathbf{k}_{1}, \lambda_{1} ; \mathbf{k}_{2}, \lambda_{2}\right\rangle & \\
& =\prod_{i} \eta_{i}\left\langle\mathbf{k}_{1}^{\prime},-\lambda_{1}^{\prime} ; \mathbf{k}_{2}^{\prime},-\lambda_{2}^{\prime} ; \mathbf{k}_{3}^{\prime},-\lambda_{3}^{\prime}\left|T^{\dagger}\right| \mathbf{k}_{1},-\lambda_{1} ; \mathbf{k}_{2},-\lambda_{2} ;\right\rangle^{*}
\end{aligned}
$$

(For a two-body amplitude one may also use reflection invariance with respect to the scattering plane.) Let us now assume that particle 1 is a spin $1 / 2$ one, say an electron. Then, ignoring all kinematical variables, the cross section for particle 1 being in a definite helicity state is

$$
\sigma( \pm)=\sum_{\left\{\lambda_{i}, \lambda_{i}^{\prime}\right\}}\left|T_{\lambda_{1}^{\prime}, \lambda_{2}^{\prime}, \lambda_{3}^{\prime} ; \pm, \lambda_{2}}\right|^{2}
$$

The difference of these cross sections may be written as

$$
\begin{array}{r}
\sigma(+)-\sigma(-)=\Re e \sum_{\left\{\lambda_{i}, \lambda_{i}^{\prime}\right\}}\left[T_{\lambda_{1}^{\prime}, \lambda_{2}^{\prime}, \lambda_{3}^{\prime} ;+, \lambda_{2}}+\prod_{i} \eta_{i} T_{-\lambda_{1}^{\prime},-\lambda_{2}^{\prime}-\lambda_{3}^{\prime} ;-,-\lambda_{2}}^{*}\right] \\
\times\left[T_{\lambda_{1}^{\prime}, \lambda_{2}^{\prime}, \lambda_{3}^{\prime} ;+, \lambda_{2}}^{*}-\prod_{i} \eta_{i} T_{-\lambda_{1}^{\prime},-\lambda_{2}^{\prime},-\lambda_{3}^{\prime} ;-,-\lambda_{2}}\right] .
\end{array}
$$

If there would be no absorptive part of $T$, i. e., $T=T^{\dagger}$, then, according to (6.3), the difference of the two helicity cross sections and hence the electron asymmetry (6.1), is zero. Therefore, the asymmtry measures the non-trivial phase as we claimed above. 
Specifying this result to the VC contribution, we find for the difference of the two cross sections (using the notation of Sect. II and dropping the kinematical factors)

$$
\begin{aligned}
\sigma(+) & -\sigma(-)=-8 \sqrt{\frac{\varepsilon}{1-\varepsilon}} \sin \phi \\
& \times \Im m\left[\Phi_{9}^{*}\left(\Phi_{1}-\Phi_{7}\right)+\Phi_{10}^{*}\left(\Phi_{2}+\Phi_{8}\right)+\Phi_{11}^{*}\left(\Phi_{3}-\Phi_{5}\right)+\Phi_{12}^{*}\left(\Phi_{4}+\Phi_{6}\right)\right]
\end{aligned}
$$

Thus, we see that $A_{L}^{V C}$ measures the imaginary part the longitudinal-transverse interference term whereas $\sigma_{L T}$ (see Eq. (2.18) ) measures its real part. In other words, $A_{L}^{V C}$ measures the relative phase between the longitudinal and transverse $\mathrm{VC}$ helicity amplitudes. It turns out, however, that the diquark model predicts only very small values for $A_{L}^{V C}$. The ultimate reason is that the longitudinal helicity amplitudes are much smaller than the transverse ones for VC scattering (see Fig. (4). So the asymetry is essentially due to the BH-VC interference where the transverse VC amplitudes do contribute, thereby producing a sizable asymetry. This asymetry is mostly due to the transverse VC amplitudes because, if we switch off the longitudinal VC amplitudes, the result changes by only a few percent.

Another possibility to measure the non-trivial perturbative phase is offered by the proton. One may measure the proton asymmetry or the polarization of the (outgoing) proton in either the electroproduction of photons or (real or virtual) Compton scattering. For the latter two-body processes this asymmetry or polarization is related to the imaginary part of the products of helicity flip and non-flip amplitudes as is well-known. The predictions from the diquark model for the proton polarization in the case of real Compton scattering is discussed by Kroll, Schürmann and Schweiger [25].

In Figs. 7 we show the electron asymmetry at a beam energy of $10 \mathrm{GeV}$ and $s=5 \mathrm{GeV}^{2}$ for several values of $Q^{2}$ and of the azimuthal angle. $A_{L}$ is generally very small but in regions of strong $\mathrm{BH}$ contamination it is sometimes spectacularly enhanced. Finally in Fig. 8 we present results for the electron asymmetry for the actual kinematical situation at $\operatorname{CEBAF}\left(s=5 \mathrm{GeV}^{2} \quad k_{0 L}=10 \mathrm{GeV}^{2}, Q^{2}=1 \mathrm{GeV}^{2}\right)$. As can be seen from that figure the asymmetry is large in our model for small values of $|\cos \theta| \quad(\simeq 0.2)$ and values of the azimuthal angle around $30^{\circ}$. The magnitude of the effect is very sensitive to details of the model and, therefore, should not be taken literally. Despite of this one may take our results as an example of what may happen. In so far we believe that is urgent and important to explore that phenomenon because it will elucidate strikingly the underlying dynamics of the electroproduction of photons in a kinematical situation which can be considered as a (fairly) hard scattering region.

\section{CONCLUDING REMARKS}

We have calculated VC scattering off protons within the framework of the diquark model which represents a particular version of the Brodsky-Lepage model appropriate for moderately large momentum transfer. The diquark model combines perturbative QCD with nonperturbative elements - the diquarks which represent quark-quark correlations in the proton wave function modeled as quasi-elementary constituents. Predictions for the VC-scattering cross section and for the $e p \rightarrow e p \gamma$ cross section are presented for kinematical situations 
accessible at CEBAF and, perhaps, in the future at an high energy accelerator like ELFE. It is also shown that the diquark model predicts a cross section for real Compton scattering in fair agreement with the data. We have also elaborated on the $\mathrm{BH}$ contamination of the electroproduction of photons which, according to the diquark model, become sizeable for small azimuthal angles. The $\mathrm{BH}$ contribution offers also the interesting possibility of measuring the relative phases between the $\mathrm{VC}$ and the $\mathrm{BH}$ amplitudes. The phases of the $\mathrm{VC}$ amplitudes are a non-trivial phenomenon generated by the fact that some of the internal quarks, diquarks and gluons may go on mass shell (note that the ingoing and outgoing quarks and diquarks forming the protons are always on mass shell). The electron asymmetry $A_{L}$ is particularly sensitive to the relative phases and can in principle be measured at CEBAF. The polarization of the outgoing nucleon in (real as well as in virtual) Compton scattering is also a measure for the relative phases between flip and non-flip helicity amplitudes.

A few words about the self-consistency of our calculations within perturbative QCD are in order. As can be seen from (A9) the running coupling constant $\alpha_{s}$ diverges in the endpoint regions $x_{1}, y_{1} \rightarrow 0,1$. This is not characteristic of the diquark model, the same happens in the pure quark HSA. As a consequence, perturbation theory looses its meaning as a weakcoupling expansion. This has previously been pointed out by Isgur and Llewellyn-Smith [34] in their analysis of form factors. One way out - actually the one we employ - is to freeze the running coupling constant once it has reached a certain value (0.5 in the case at hand) 355. In addition we make use of DAs which strongly suppress the end-point regions. The freezing of $\alpha_{s}$ introduces a new external parameter. The modified HSA proposed by Sterman and Li [15], in which the transverse momenta of the constituents as well as Sudakov corrections are taken into account, avoids the introduction of such an external parameter. The Sudakov corrections select components of the wave functions with small spatial separations of the constituents. With increasing momentum transfer the allowed spatial separations are getting smaller and smaller. Asymptotically, only those components remain which are taken into account in the standard HSA of Brodsky and Lepage [7]. The numerical effect of the Sudakov suppressions is similar to that of freezing in $\alpha_{s}$. This has been demonstrated recently in [15 18] for electromagnetic form factors (within the pure HSA). It is claimed that in this way the self-consistency of the perturbative contributions can be reestablished for $Q$ larger than a few $\mathrm{GeV}$ without introducing a freezing parameter. We also note that the transverse momentum dependence of the hadronic wave function helps in achieving self-consistency at rather low values of momentum transfer [18]. In [27] an analogous calculation for the Sdiquark contributions to the proton form factor has been performed and the authors of [27] arrive at the same conclusion. A treatment of the end-point regions in the manner proposed by Li and Sterman [15] diminishes the results for the form factors obtained with the diquark model by only 10 to $20 \%$. Since Sudakov corrections mainly depend on colour and not on spin a similar behaviour for $V$ diquarks is to be expected. That small suppression can be compensated for by adjusting the parameters of the diquark model appropriately. Effects of similar magnitude are expected for Compton scattering. We consider these suppressions not explicitely but understand them as being absorbed in our parameters. For an effective model as the diquark model is, this procedure is sufficient. 


\section{APPENDIX A: HELICITY AMPLITUDES FOR $\gamma^{*} q S \rightarrow \gamma q S$}

The process $\gamma^{*} q S \rightarrow \gamma q S$ only contributes to the hadronic helicity-conserving amplitudes. This implies that 6 of the 12 independent amplitudes $\Phi_{i}^{(S, n)}$ given in (2.13) are zero

$$
\Phi_{2}^{(S, n)}=\Phi_{4}^{(S, n)}=\Phi_{6}^{(S, n)}=\Phi_{8}^{(S, n)}=\Phi_{10}^{(S, n)}=\Phi_{12}^{(S, n)}=0 .
$$

For dynamical reasons the diquark model yields also

$$
\Phi_{7}^{(S, n)}=0
$$

The remaining amplitudes $\Phi_{i}^{(S, n)}$ read in our model:

$$
\begin{aligned}
& \Phi_{1}^{(S, 3)}=\frac{4 A_{T}^{(S, 3)}}{q_{1}^{2}+i \varepsilon} \frac{s^{2}}{t u\left(s+Q^{2}\right)}\left\{\frac{x_{1} s}{x_{2} y_{2}}-\frac{1}{y_{1} u-y_{2} Q^{2}}\left(\frac{u Q^{2}}{y_{1} y_{2}}-\left(u+Q^{2}\right)^{2}\right)\right\} \\
& \Phi_{1}^{(S, 4)}=4 A_{T}^{(S, 4)} \frac{1}{u}\left\{\frac{\left(s+Q^{2}\right)}{q_{1}^{2}+i \varepsilon} \frac{\left(s+x_{2} t\right)}{g_{4 a}^{2}+i \varepsilon}-\frac{1}{g_{4 b}^{2}+i \varepsilon}\left(t+\frac{u}{y_{1}}\right)\right\} \\
& \Phi_{1}^{(S, 5)}=4 A_{T}^{(S, 5)} \frac{1}{x_{1} y_{1} u} \\
& \Phi_{3}^{(S, 3)}=\frac{4 A_{T}^{(S, 3)}}{y_{1} u-y_{2} Q^{2}} \frac{Q^{2}}{u}\left\{\frac{y_{1} u}{x_{2} y_{2}\left(s+Q^{2}\right)}+\frac{1}{q_{1}^{2}+i \varepsilon}\left(s+Q^{2}-\frac{s Q^{2}}{x_{1} x_{2}\left(s+Q^{2}\right)}\right)\right\} \\
& \Phi_{3}^{(S, 4)}=\frac{4 A_{T}^{(S, 4)}}{y_{2} u-y_{1} Q^{2}} \frac{t Q^{2}}{u\left(s+Q^{2}\right)}\left\{\frac{1}{x_{1}}+\frac{u+y_{2} t}{g_{4 a}^{2}+i \varepsilon}\right\} \\
& \Phi_{3}^{(S, 5)}=-\frac{4 A_{T}^{(S, 5)}}{y_{2} u-y_{1} Q^{2}} \frac{Q^{2}\left(s+Q^{2}+y_{2} t\right)}{x_{1} y_{1} u\left(s+Q^{2}\right)} \\
& \Phi_{5}^{(S, 3)}=-\frac{4 A_{T}^{(S, 3)}}{y_{1} u-y_{2} Q^{2}} \frac{s}{t}\left\{\frac{y_{1} u}{x_{2} y_{2}\left(s+Q^{2}\right)}+\frac{1}{q_{1}^{2}+i \varepsilon}\left(s+Q^{2}-\frac{s Q^{2}}{x_{1} x_{2}\left(s+Q^{2}\right)}\right)\right\} \\
& \Phi_{5}^{(S, 4)}=\frac{4 A_{T}^{(S, 4)}}{y_{2} u-y_{1} Q^{2}}\left\{-\frac{s}{x_{1}\left(s+Q^{2}\right)}-\frac{\left(u+y_{2} t\right)}{s+Q^{2}} \frac{s}{g_{4 a}^{2}+i \varepsilon}+\frac{x_{2} s}{x_{1}} \frac{1}{g_{4 b}^{2}+i \varepsilon}\right\} \\
& \Phi_{5}^{(S, 5)}=\frac{4 A_{T}^{(S, 5)}}{y_{2} u-y_{1} Q^{2}} \frac{y_{2} s}{x_{1} y_{1} u\left(s+Q^{2}\right)} \\
& \Phi_{9}^{(S, 3)}=0 \\
& \Phi_{9}^{(S, 4)}=2 A_{0}^{(S, 4)} \frac{y_{2} s}{\left(g_{4 a}^{2}+i \varepsilon\right)\left(D_{1}^{2}+i \varepsilon\right)} \\
& \Phi_{9}^{(S, 5)}=-2 A_{0}^{(S, 5)} \frac{s}{x_{1} y_{1} t} \frac{1}{D_{1}^{2}+i \varepsilon} \\
& \Phi_{12}^{(S, 3)}=-\frac{4 A_{0}^{(S, 3)}}{y_{1} u-y_{2} Q^{2}} \frac{s}{t}\left\{\frac{y_{1} u}{x_{2} y_{2}\left(s+Q^{2}\right)}+\frac{1}{q_{1}^{2}+i \varepsilon}\left(s+Q^{2}-\frac{s Q^{2}}{x_{1} x_{2}\left(s+Q^{2}\right)}\right)\right\} \\
& \Phi_{12}^{(S, 4)}=\frac{2 A_{0}^{(S, 4)}}{y_{2} u-y_{1} Q^{2}}\left\{-\frac{2 s}{x_{1}\left(s+Q^{2}\right)}+2\left(1+\frac{y_{1} t}{s+Q^{2}}\right) \frac{s^{2}}{g_{4 a}^{2}+i \varepsilon}+\frac{x_{2} s}{x_{1}} \frac{1}{g_{4 b}^{2}+i \varepsilon}\right\}
\end{aligned}
$$




$$
\Phi_{12}^{(S, 5)}=\frac{2 A_{0}^{(S, 5)}}{y_{2} u-y_{1} Q^{2}} \frac{s}{t} \frac{s+Q^{2}+2 y_{2} t}{x_{1} y_{1}\left(s+Q^{2}\right)}
$$

where

$$
\begin{aligned}
& A_{T}^{(S, n)}=\sqrt{-\frac{u}{s}} \alpha_{s}\left(g_{n}^{2}\right) F_{S}^{(n)}\left(Q_{n}^{2}\right) \\
& A_{0}^{(S, n)}=\frac{\sqrt{-2 t Q^{2}}}{s} \alpha_{s}\left(g_{n}^{2}\right) F_{S}^{(n)}\left(Q_{n}^{2}\right) .
\end{aligned}
$$

For $Q^{2}=0$ the $\Phi_{3}^{(S, n)}$ are zero and the remaining amplitudes agree with those quoted by Kroll, Schürmann and Schweiger [25]. The various gluon propagators appearing as arguments in $\alpha_{s}$ and as arguments in the various diquark vertex functions are taken as:

$$
\begin{array}{lll}
g_{3}^{2}=-x_{2} y_{2} t & g_{4}^{2}=-\frac{1}{2}\left(x_{1} y_{2}+x_{2} y_{1}\right) t & g_{5}^{2}=-x_{1} y_{1} t \\
Q_{3}^{2}=g_{3}^{2} & Q_{4}^{2}=g_{4}^{2}+Q_{3}^{2} & Q_{5}^{2}=g_{5}^{2}+Q_{3}^{2}
\end{array}
$$

$g_{4 a}, g_{4 b}, q_{1}$ and $D_{1}$ are the momenta of the internal gluon, quark and diquarks which lead to the poles within the range of integration. The corresponding virtualities are

$$
\begin{array}{ll}
q_{1}^{2}=x_{1} s-x_{2} Q^{2} & g_{4 a}^{2}=x_{1} y_{2} s+x_{2} y_{1} u-x_{2} y_{2} Q^{2} \\
D_{1}^{2}=x_{2} s-x_{1} Q^{2} & g_{4 b}^{2}=x_{2} y_{1} s+x_{1} y_{2} u-x_{1} y_{1} Q^{2}
\end{array}
$$

The propagator poles are integrable and do not destroy the validity of the hard scattering approach [29]. The poles are handled in the usal way by using the $i \varepsilon$ prescription

$$
\frac{1}{x \pm i \epsilon}=P\left(\frac{1}{x}\right) \mp i \pi \delta(x)
$$

In the case that there is only one pole in the region of integration, the integrals over $x_{1}$ can be performed in the following way:

$$
\begin{aligned}
& \int_{0}^{1} d x_{1} \frac{f\left(x_{1}, y_{1}, s, t, Q^{2}\right)}{p^{2}+i \varepsilon} \phi_{D}\left(x_{1}\right) \\
& =\int_{0}^{1} d x_{1} \frac{f\left(x_{1}, y_{1}, s, t, Q^{2}\right) \phi_{D}\left(x_{1}\right)-f\left(x_{1}^{(p)}, y_{1}, s, t, Q^{2}\right) \phi_{D}\left(x_{1}^{(p)}\right)}{p^{2}} \\
& +f\left(x_{1}^{(p)}, y_{1}, s, t, Q^{2}\right) \phi_{D}\left(x_{1}^{(p)}\right)\left(P . V \cdot \int_{0}^{1} \frac{d x_{1}}{p^{2}}-i \pi\left|\frac{\partial p^{2}}{\partial x_{1}}\right|^{-1}\right) .
\end{aligned}
$$

where $x_{1}^{(p)}$ is the zero of the equation $p^{2}=0$ and the different $p^{2}$ are given in Eq. (A10). The necessary principal value integral over $x_{1}$ is then carried out analytically. The different integrals and the derivatives of the propagators are listed in Table I. In cases when two propagators go on shell at the same time one has to make a partial fractioning before applying (A12). 


\section{REFERENCES}

[1] A. F. Sill et al., Phys. Rev. D48 (1993) 29; R. G. Arnold et al., Phys. Rev. Lett. 57 (1986) 174.

[2] P. E. Bosted et al., Phys. Rev. Lett. 68 (1992) 3841.

[3] M. A. Shupe et al., Phys. Rev. D19 (1979) 1921.

[4] C. Audit et al., CEBAF proposal PR-92-050 (1993).

[5] J. Arvieux and E. de Sanctis, The ELFE project, Italian Physical Society Conference Proceedings 44 (1993).

[6] L. W. Mo and Y. S. Tsai, Rev. of Mod. Phys. 41 (1969) 205.

[7] S. J. Brodsky and G. P. Lepage, Phys. Rev. D22 (1980) 2157.

[8] A. H. Mueller, Phys. Rep. 73 (1981) 237.

[9] V. L. Chernyak and A. R. Zhitnitsky, Phys. Rep. 112 (1984) 173.

[10] V. L. Chernyak and I. R. Zhitnitsky, Nucl. Phys. B246 (1984) 52; V. L. Chernyak, A. Ogloblin and I. R. Zhitnitsky, Z. Phys. C42 (1989) 569.

[11] I. D. King and C. T. Sachrajda, Nucl. Phys. B279 (1987) 785.

[12] A. S. Kronfeld and D. M. Photiadis, Phys. Rev. D31 (1985) 2939; S. Gottlieb and A. S. Kronfeld, Phys. Rev. Lett. 55 (1985) 2531; Phys. Rev. D33 (1986) 227.

[13] Y. G. Richards, C. T. Sachrajda and C. J. Scott, Nucl. Phys. B286 (1987) 683; G. Martinelli and C. T. Sachrajda, Phys. Lett. B217 (1989) 319.

[14] M. Bergmann and N. G. Stefanis, Phys. Rev. D48 (1993) R2990.

[15] H. N. Li and G. Sterman, Nucl. Phys. B381 (1992) 129.

[16] H. N. Li, Phys. Rev. D48 (1993) 4243.

[17] J. Bolz, R. Jakob, P. Kroll, M. Bergmann and N. G. Stefanis, Z. Phys. C66 (1995) 267 and Phys. Lett. B342 (1995) 345.

[18] R. Jakob and P. Kroll, Phys. Lett. B315 (1993) 463; B319 (1993) 545(E).

[19] B. Nižić, Phys. Rev. D35 (1987) 80.

[20] D. G. Crabb et al., Phys. Rev. Lett. 65 (1990) 3241; P. R. Cameron et al., Phys. Rev. D32 (1985) 3070; P. H. Hansen et al., Phys. Rev. Lett. 50 (1983) 802; D. C. Peaslee et al., Phys. Rev. Lett. 51 (1983) 2359; J. Antille et al., Nucl. Phys. B185 (1981) 1.

[21] S. Saroff et al., Phys. Rev. Lett. 64 (1990) 995.

[22] P. Kroll, Proceedings of the 8th Intern. Symp. on High Energy Spin Physics, Minneapolis (1988).

[23] D. Sivers, Phys. Rev. D41 (1990) 83.

[24] M. Anselmino, P. Kroll and B. Pire, Z. Phys. C36 (1987) 89.

[25] P. Kroll, Proceedings of the Adriatico Research Conference on Spin and Polarization Dynamics in Nuclear and Particle Physics, Trieste, Italy, 1988; P. Kroll and W. Schweiger, Nucl. Phys. A474 (1987) 608; P. Kroll, B. Quadder and W. Schweiger, Nucl. Phys. B316 (1989) 373; P. Kroll, W. Schweiger and M. Schürmann, Int. Jour. of Mod. Physics A6 (1991) 4107 and Z. Phys. A338 (1991) 339.

[26] P. Kroll, Th. Pilsner, M. Schürmann and W. Schweiger, Phys. Lett. B316 (1993) 546.

[27] R. Jakob, P. Kroll, M. Schürmann and W. Schweiger, Z. Phys. A347 (1993) 109.

[28] G. R. Farrar, G. Sterman and H. Zhang, Phys. Rev. Lett. 62 (1989) 2229.

[29] G. R. Farrar and H. Zhang, Phys. Rev. D41 (1990) 3348.

[30] A. S. Kronfeld and B. Nižić, Phys. Rev. D44 (1991) 3445. 
[31] T. D. Lee and C. N. Yang, Phys. Rev. 128 (1962) 885.

[32] M. Virchaux and A. Milsztajn, Phys. Lett. B274 (1992) 221.

[33] Computer-algebra system FORM by J. Vermaseren Version 1.0 (1990).

[34] N. Isgur and C. H. Llewellyn-Smith, Nucl. Phys. B317 (1989) 526.

[35] J. M. Cornwall, Phys. Rev. D26 (1982) 1553. 


\section{TABLES}

TABLE I. Zeros of propagators from Eq. (A12), partial derivatives and principal value integrals.

\begin{tabular}{|c|c|c|c|}
\hline$p^{2}$ & $x_{1}^{(p)}$ & $\frac{\partial p^{2}}{\partial x_{1}}$ & P.V. $\int_{0}^{1} \frac{d x_{1}}{p^{2}}$ \\
\hline$q_{1}^{2}$ & $\frac{Q^{2}}{s+Q^{2}}$ & $s+Q^{2}$ & $\frac{1}{s+Q^{2}} \ln \left(\frac{s}{Q^{2}}\right)$ \\
\hline$D_{1}^{2}$ & $\frac{s}{s+Q^{2}}$ & $s+Q^{2}$ & $\frac{1}{s+Q^{2}} \ln \left(\frac{s}{Q^{2}}\right)$ \\
\hline$g_{4 a}^{2}$ & $\frac{y_{1} u-y_{2} Q^{2}}{y_{2} t+u}$ & $-\left(y_{2} t+u\right)$ & $\frac{-1}{y_{1} t+u} \ln \left(\frac{y_{1} u+y_{2} Q^{2}}{y_{2} t-u}\right)$ \\
\hline$g_{4 b}^{2}$ & $-\frac{y_{1} s}{y_{1} t+u}$ & $-\left(y_{1} t+u\right)$ & $\frac{-1}{y_{1} t+u} \ln \left(\frac{y_{1} s}{y_{1} t-u}\right)$ \\
\hline
\end{tabular}




\section{FIGURES}

FIG. 1. The Bethe-Heitler and the virtual Compton contributions to the process $e p \rightarrow e p \gamma$. The momenta and the helicities of the various particles appearing in that process are indicated.

FIG. 2. Feynman graphs contributing to the process $\gamma^{*} p \rightarrow \gamma p$. Graphs with the two photons interchanged are not shown.

FIG. 3. The cross section for real Compton scattering off protons scaled by $s^{6}$ vs. $\cos \theta$ for three different photon energies. The experimental data are taken from [3].

FIG. 4. The cross section for virtual Compton scattering vs. $\cos \theta$ for several values of $Q^{2} / s$ at a) $s=5 \mathrm{GeV}^{2}$, b) $10 \mathrm{GeV}^{2}$. Upper left: the transverse cross section scaled by $s^{6}$. Upper right: the ratio of the longitudinal over the transverse cross sections. Lower left (right): the ratio of the longitudinal (transverse) - transverse interference term over the transverse cross section.

FIG. 5. The cross section for virtual Compton scattering vs. $Q^{2}$. For notations see Fig. 1 .

FIG. 6. The difference of the full photon electroproduction cross section and the VC contribution to it over the full cross section vs. $\cos \theta$ for several combinations of values of the beam energy $k_{0 L}, Q^{2}$ and the azimuthal angle $\Phi$. a) $s=5 \mathrm{GeV}^{2}$, b) $10 \mathrm{GeV}^{2}$

FIG. 7. The electron asymmetry $A_{L}$ vs. $\cos \theta$ for several values of $Q^{2}$ and of the azimuthal angle.

FIG. 8. The electron asymmetry at CEBAF. Top: $A_{L}$ vs. $\phi$ for several values of $\cos \theta$. Bottom: $A_{L}$ vs. $\cos \theta$ for several values of $\phi$. 
Real compton Scattering

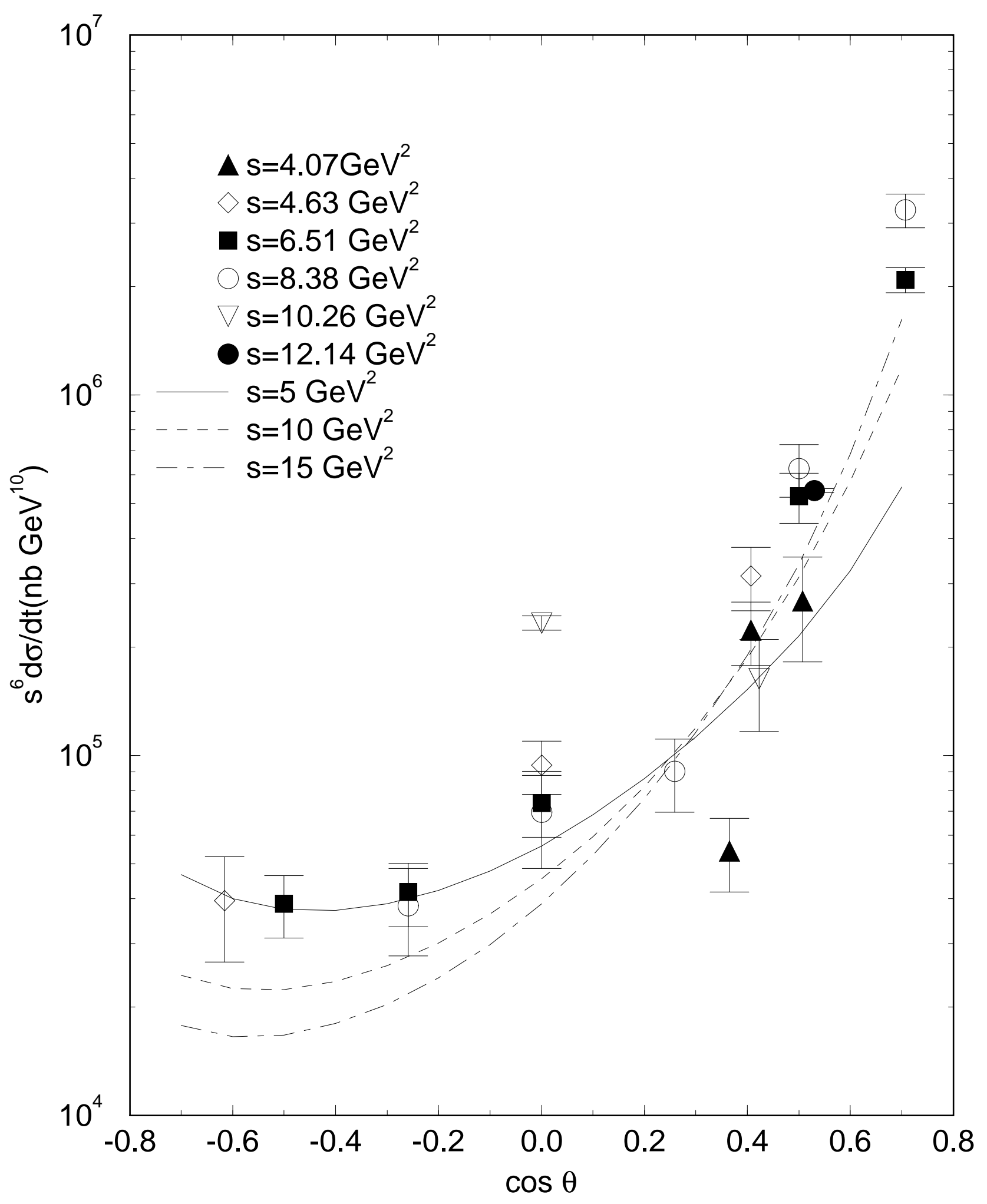




\section{$\mathrm{S}=15 \mathrm{GeV}^{2}$}
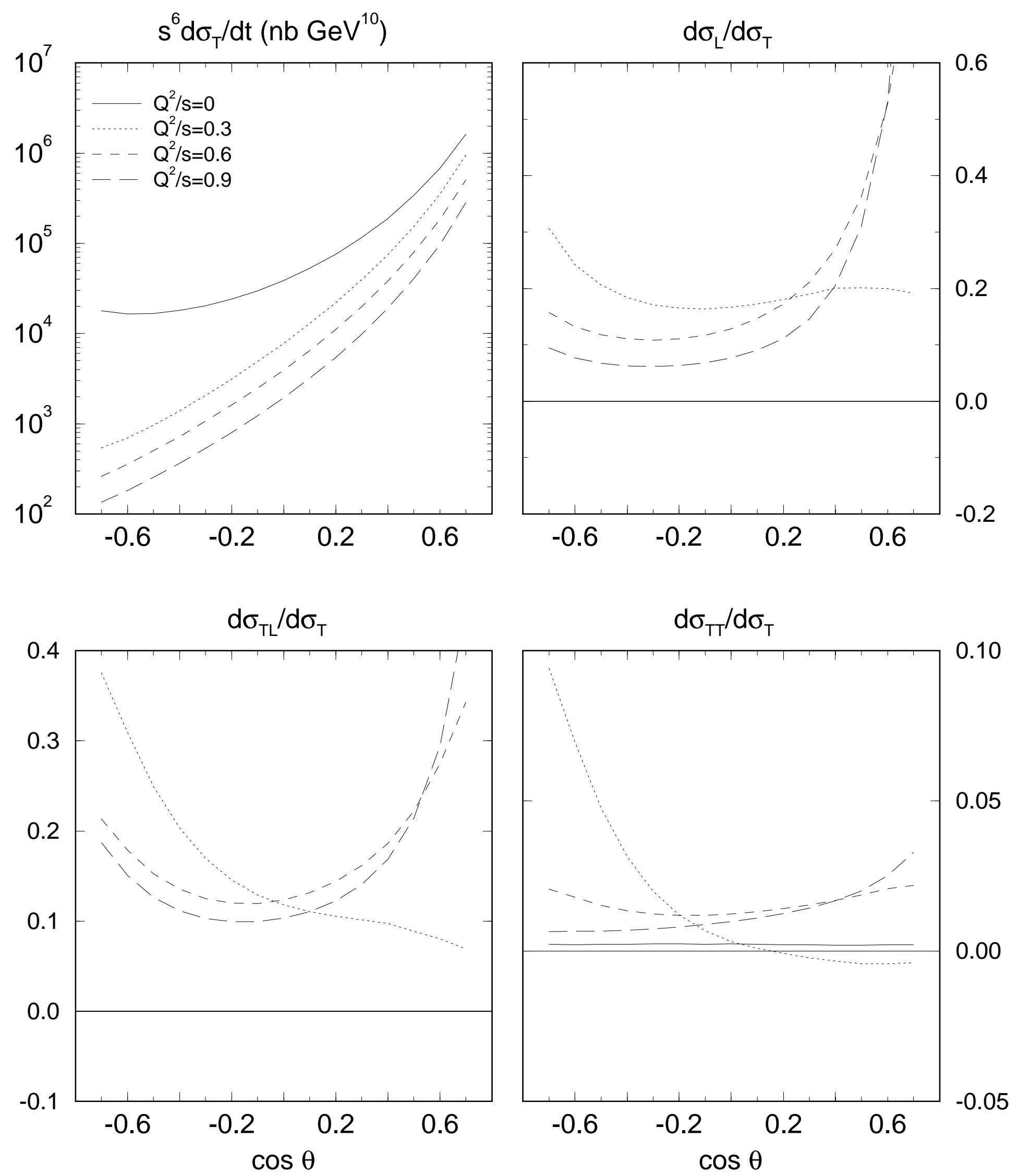
$\mathrm{s}=10 \mathrm{GeV}^{2} \quad \cos \theta=-0.6$
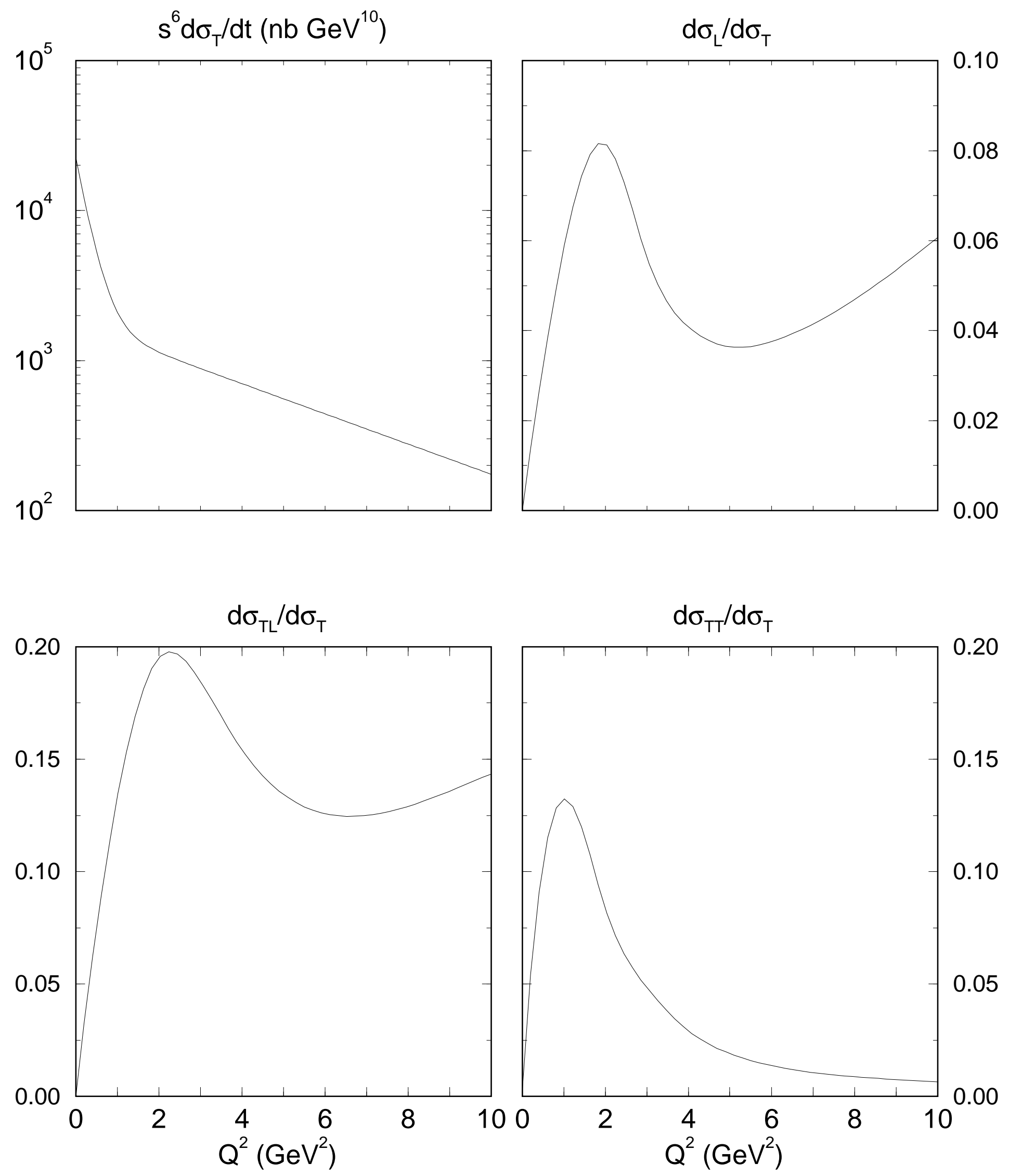

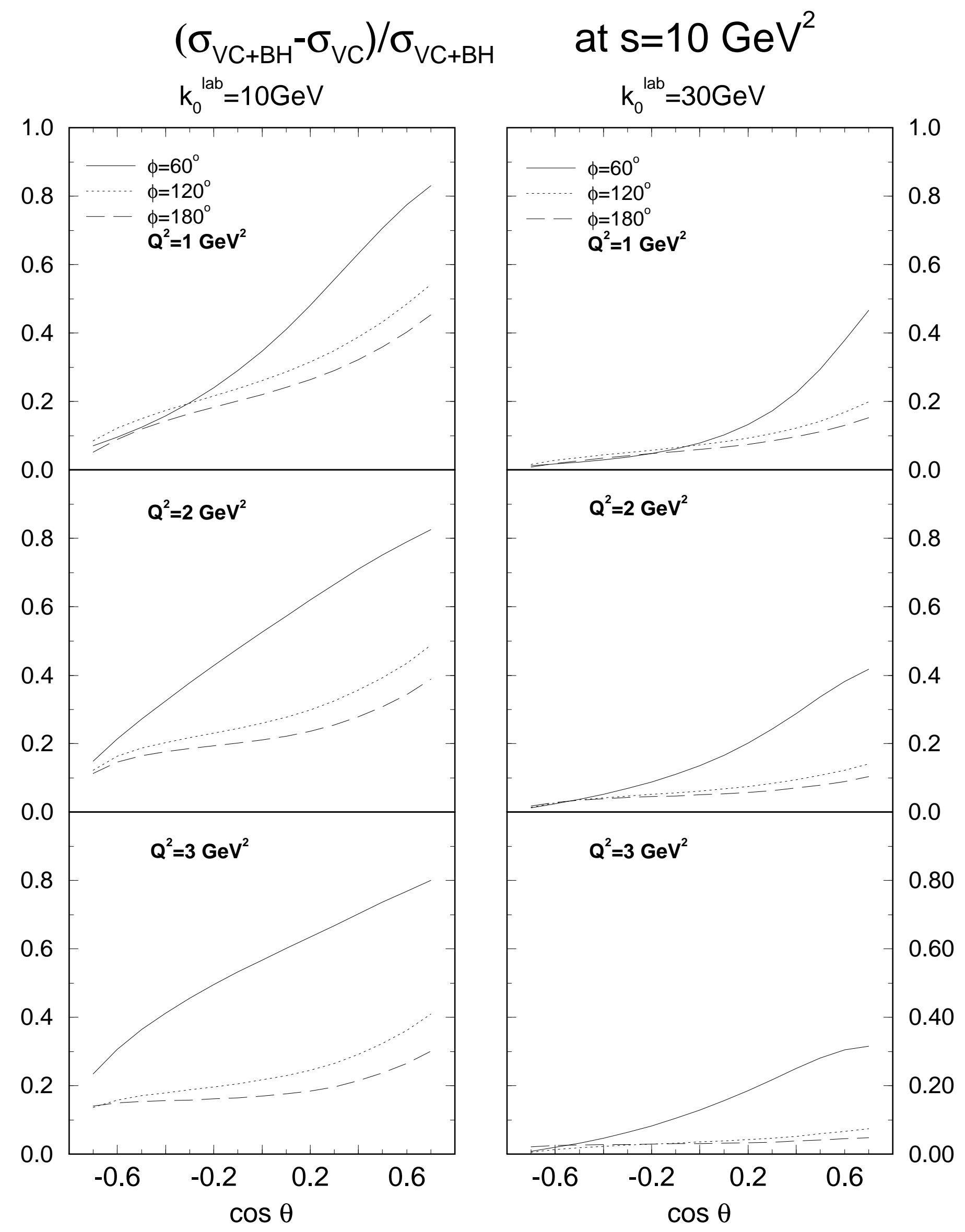


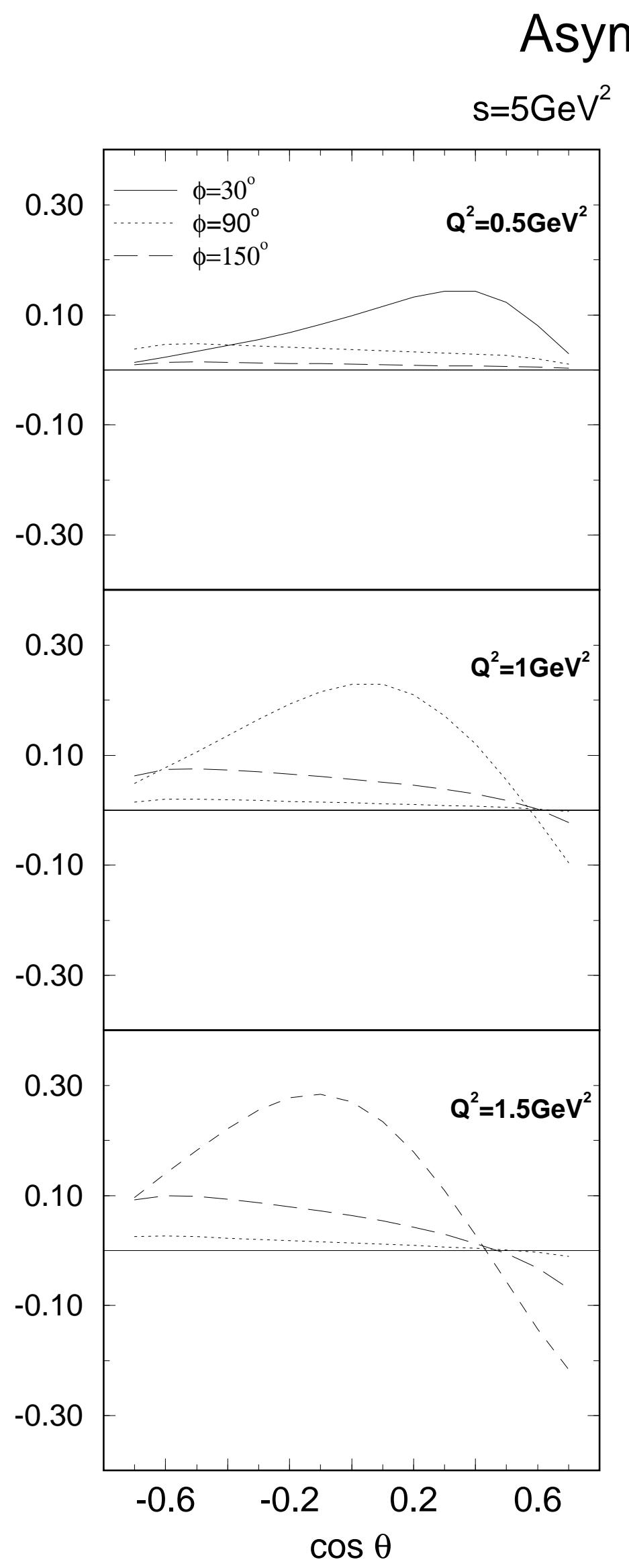

$k^{0}{ }_{\text {ab }}=10 \mathrm{GeV}$

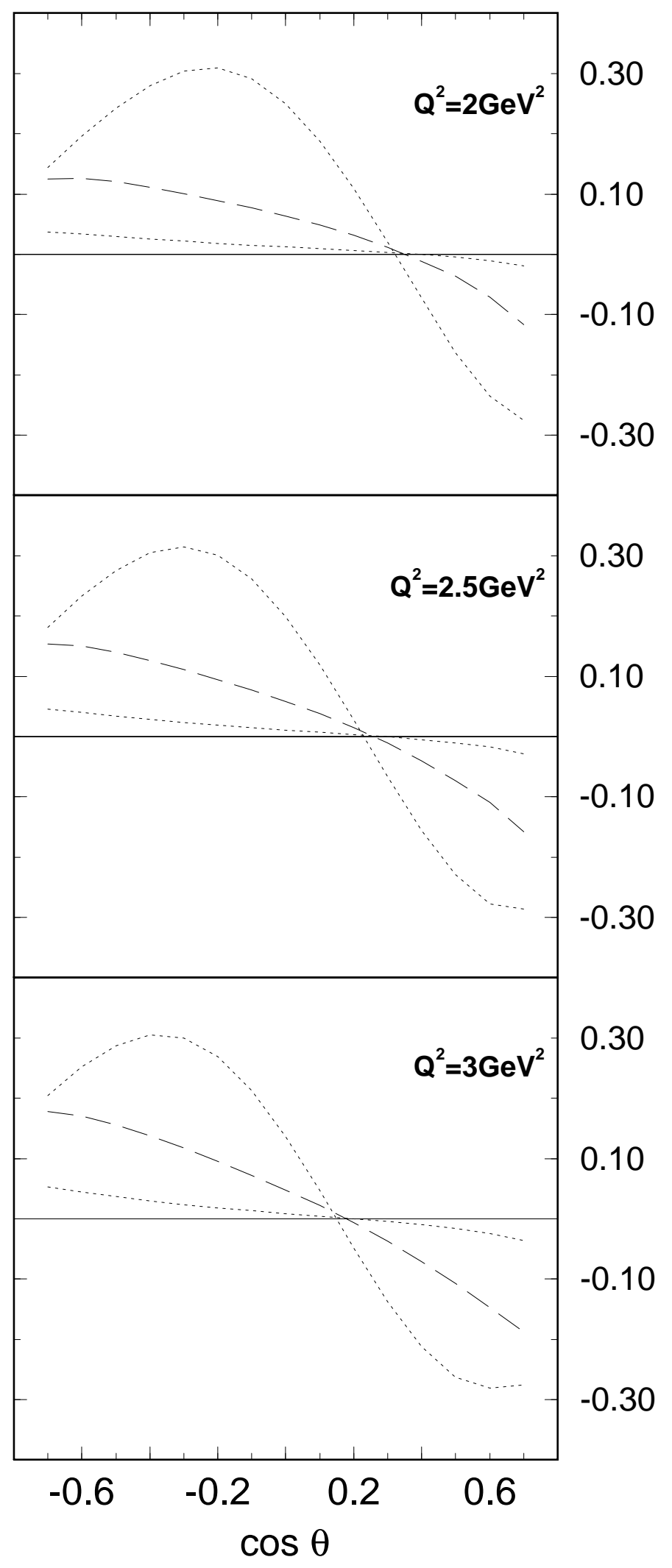




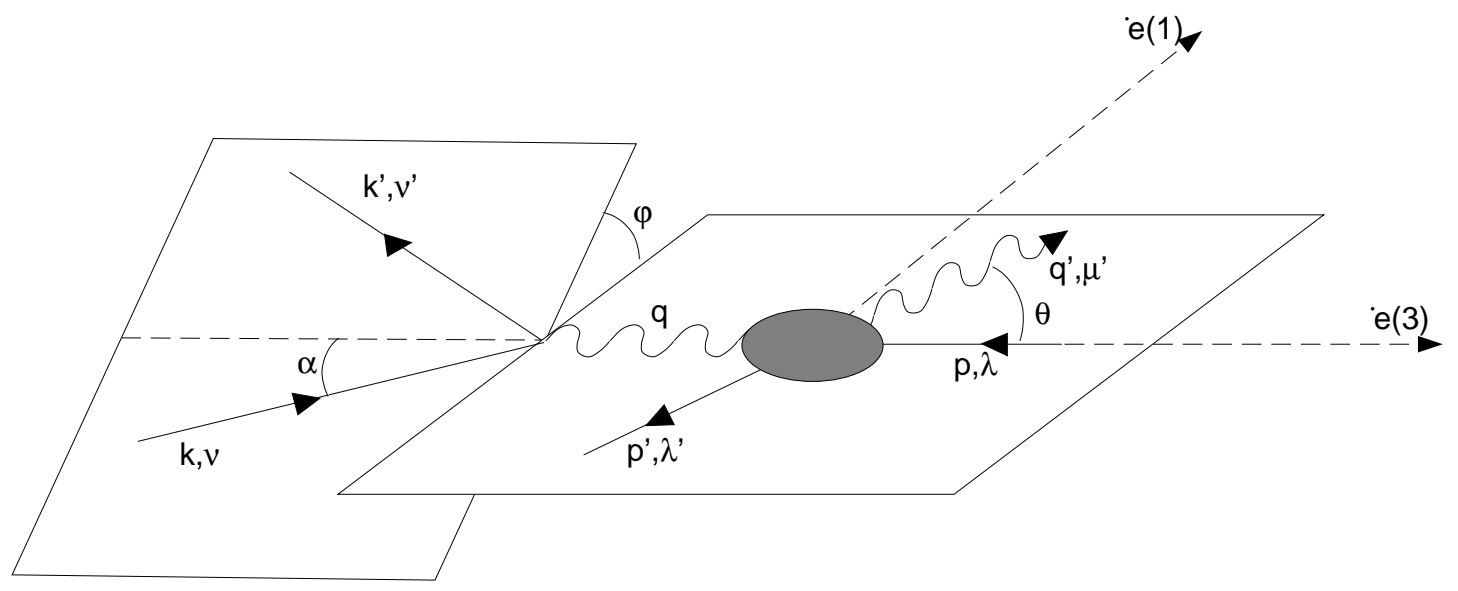



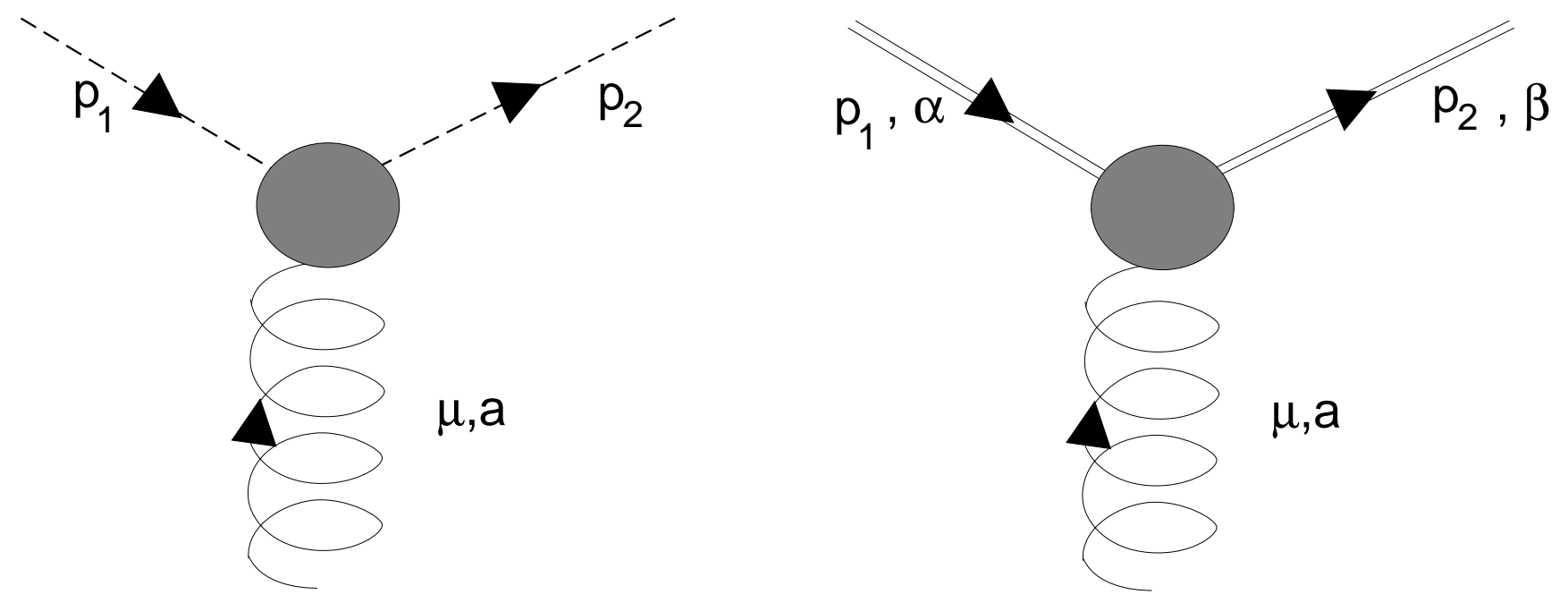


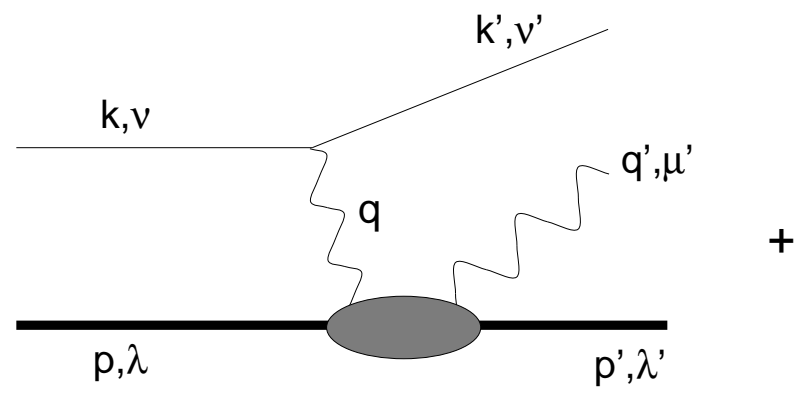

(a)

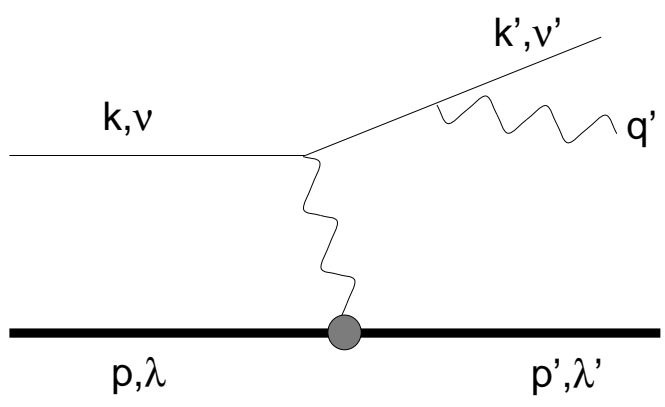

(b)

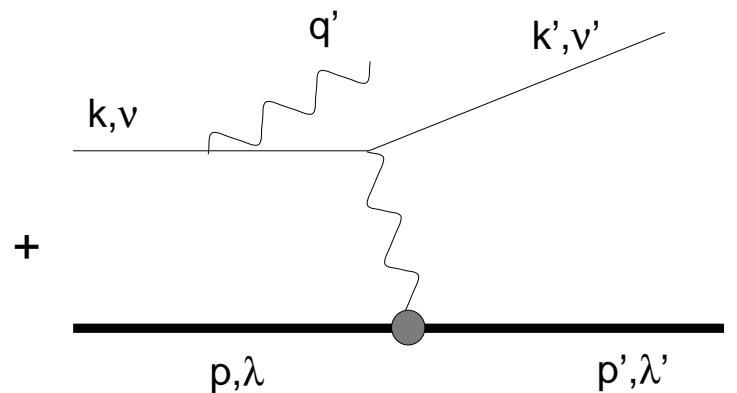

(c) 

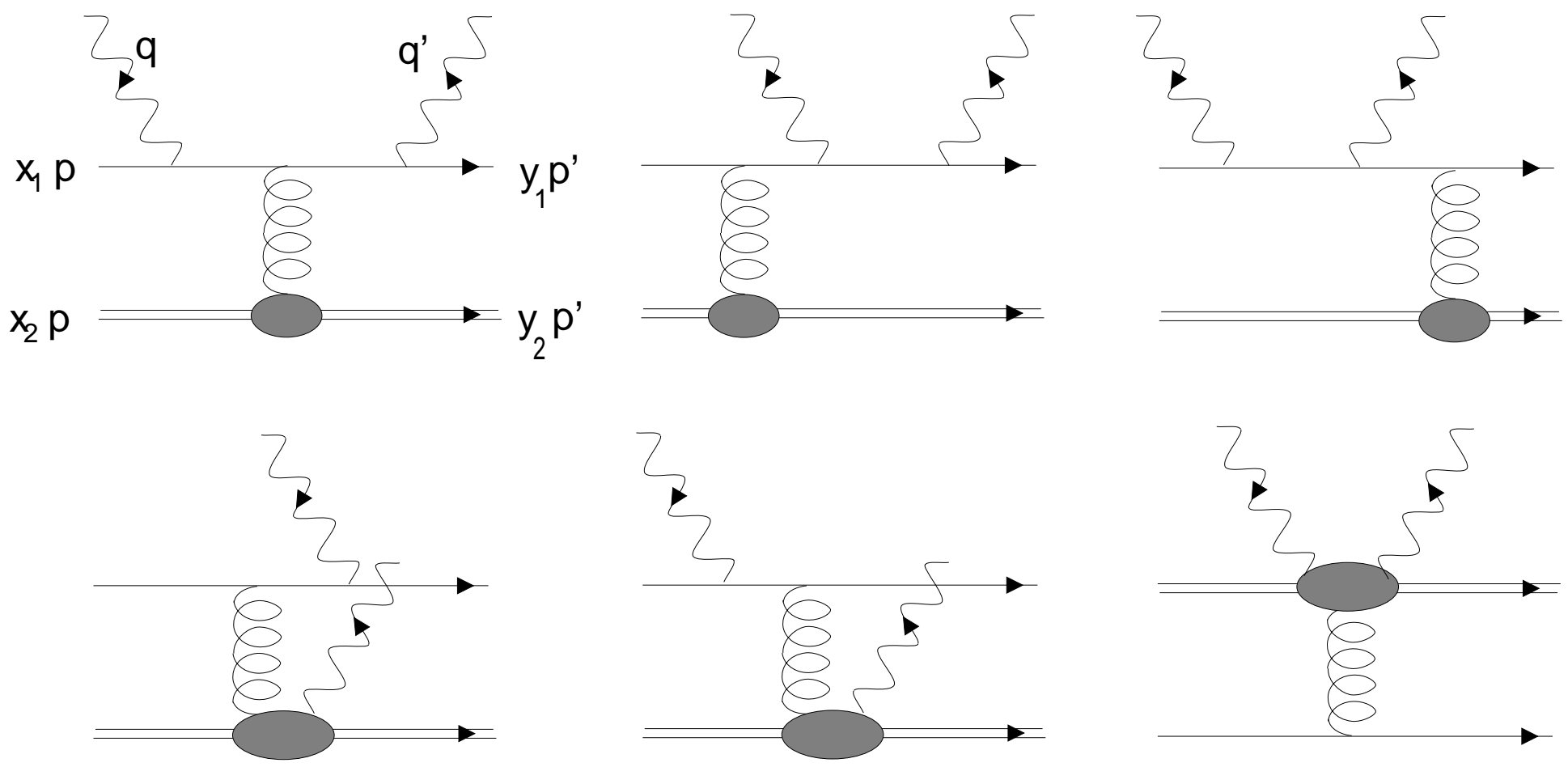
Real compton Scattering

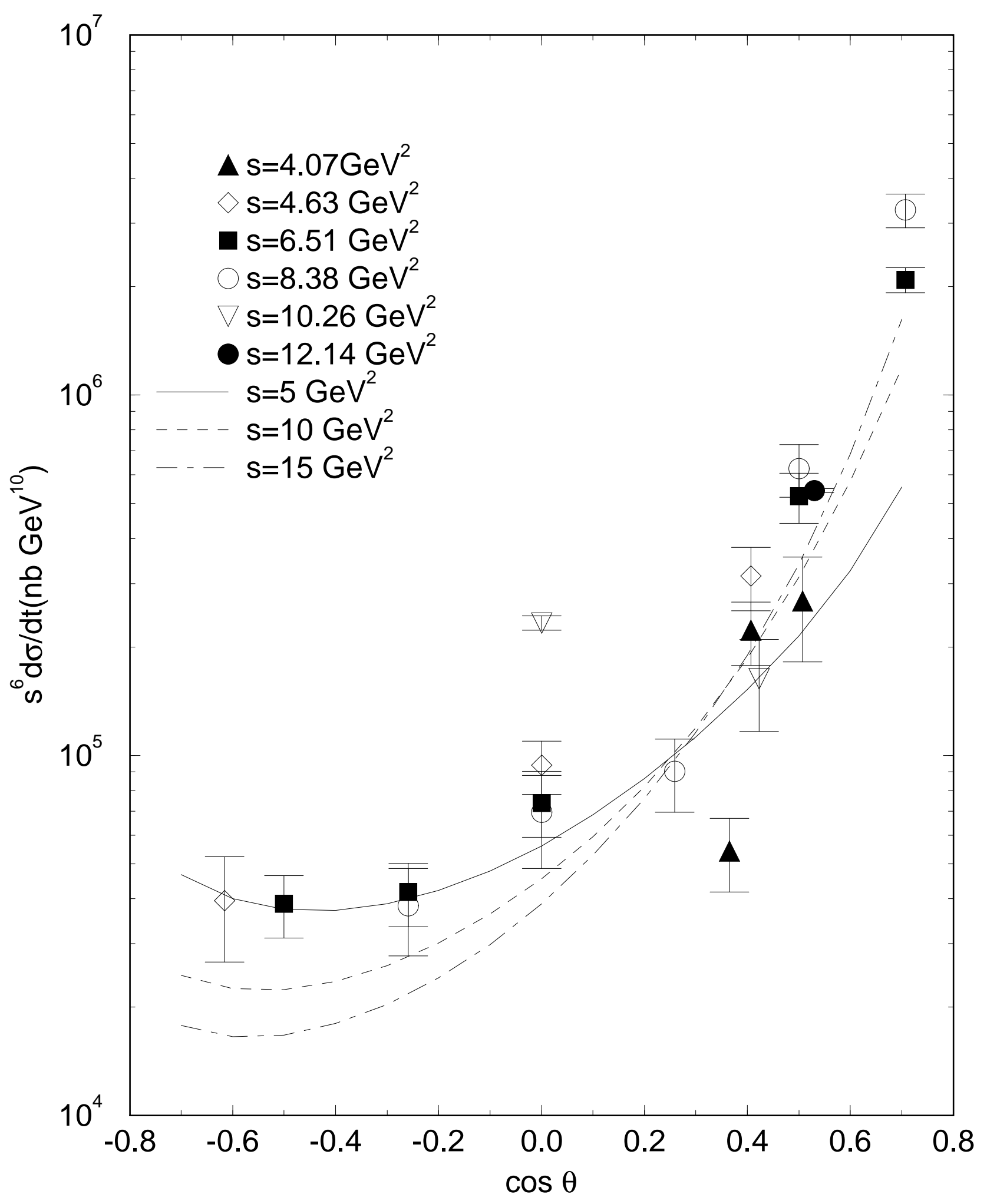




\section{$\mathrm{S}=5 \mathrm{GeV}^{2}$}
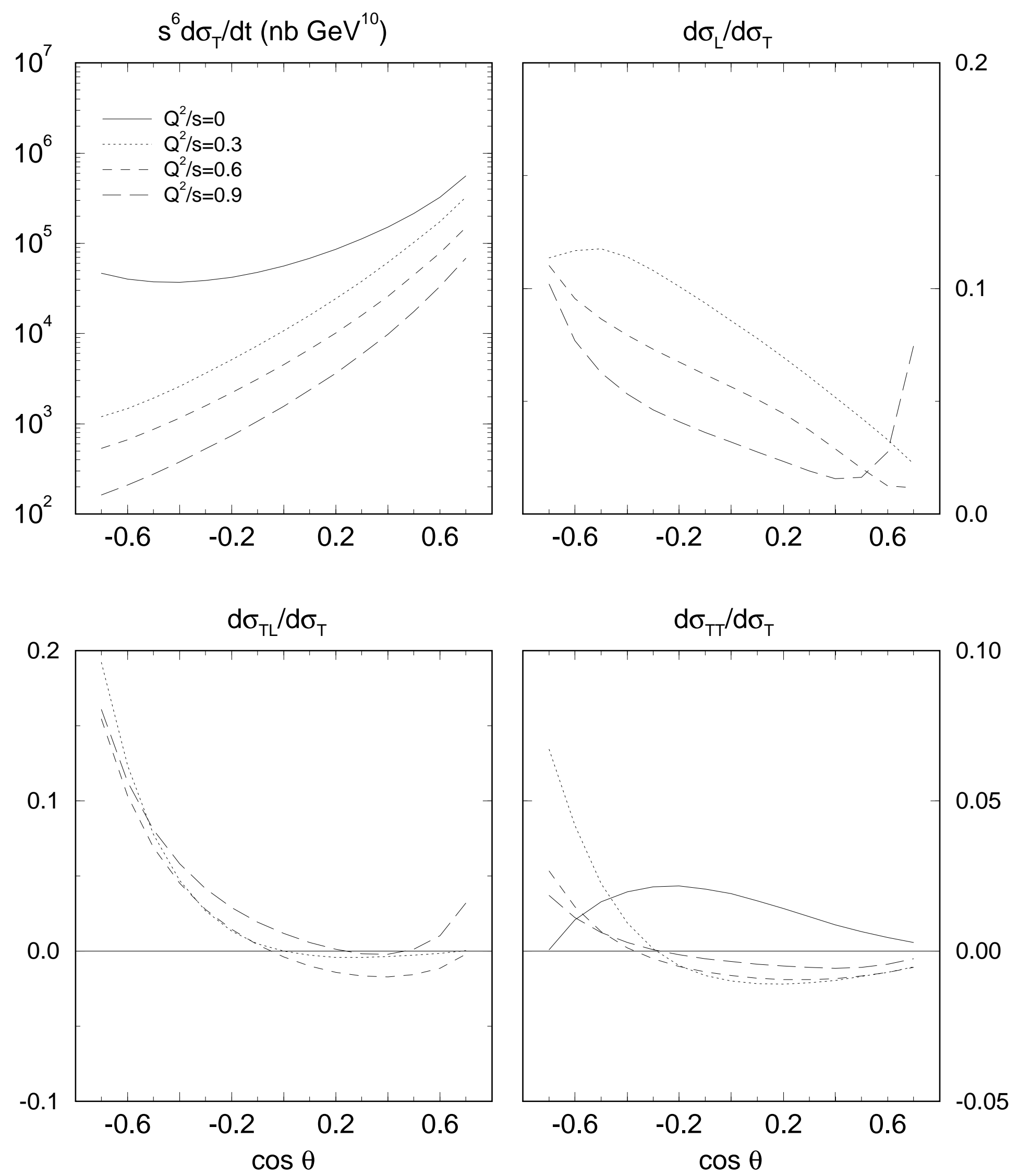


\section{$\mathrm{S}=10 \mathrm{GeV}^{2}$}
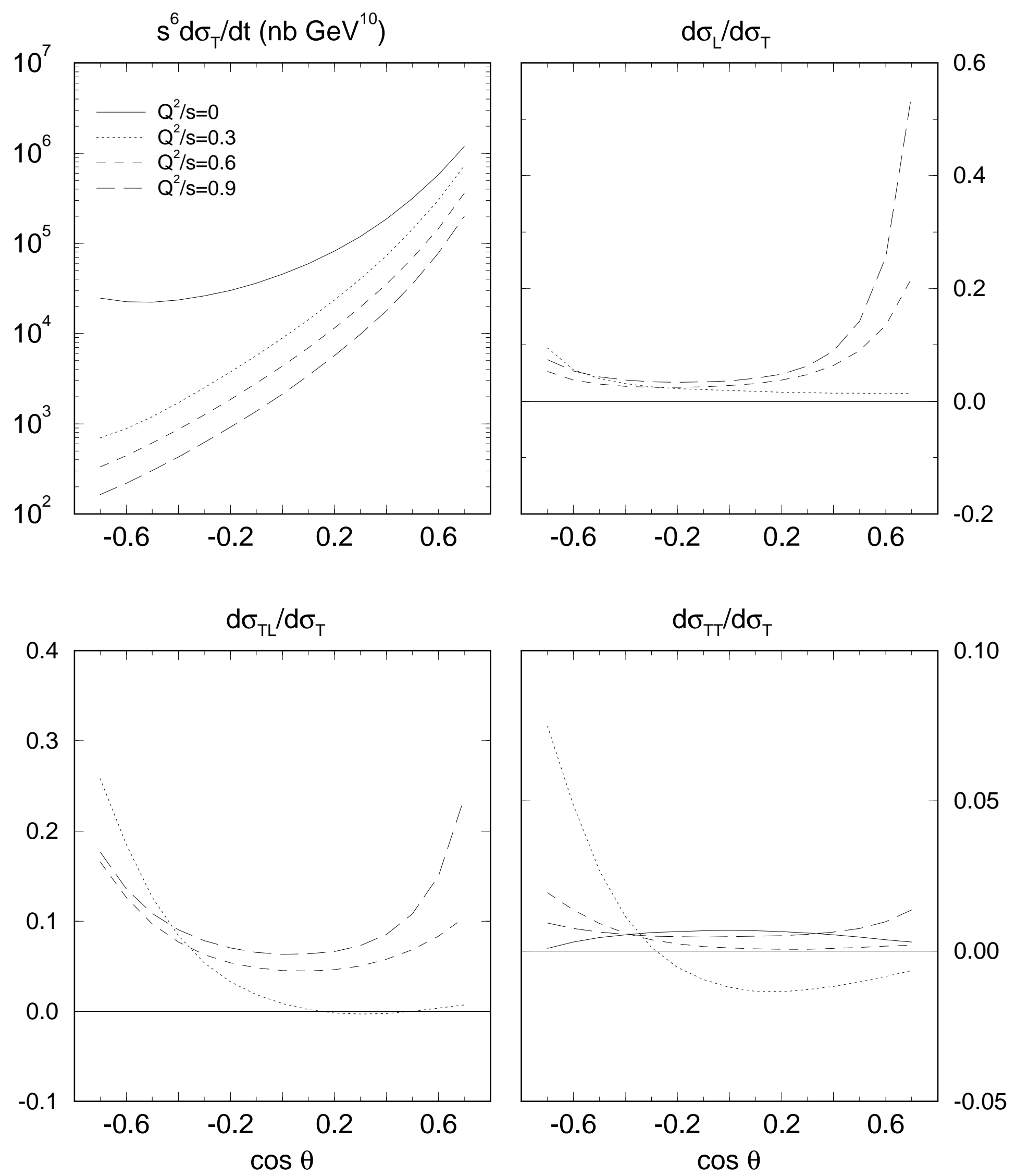
$\mathrm{s}=10 \mathrm{GeV}^{2} \quad \cos \theta=-0.6$
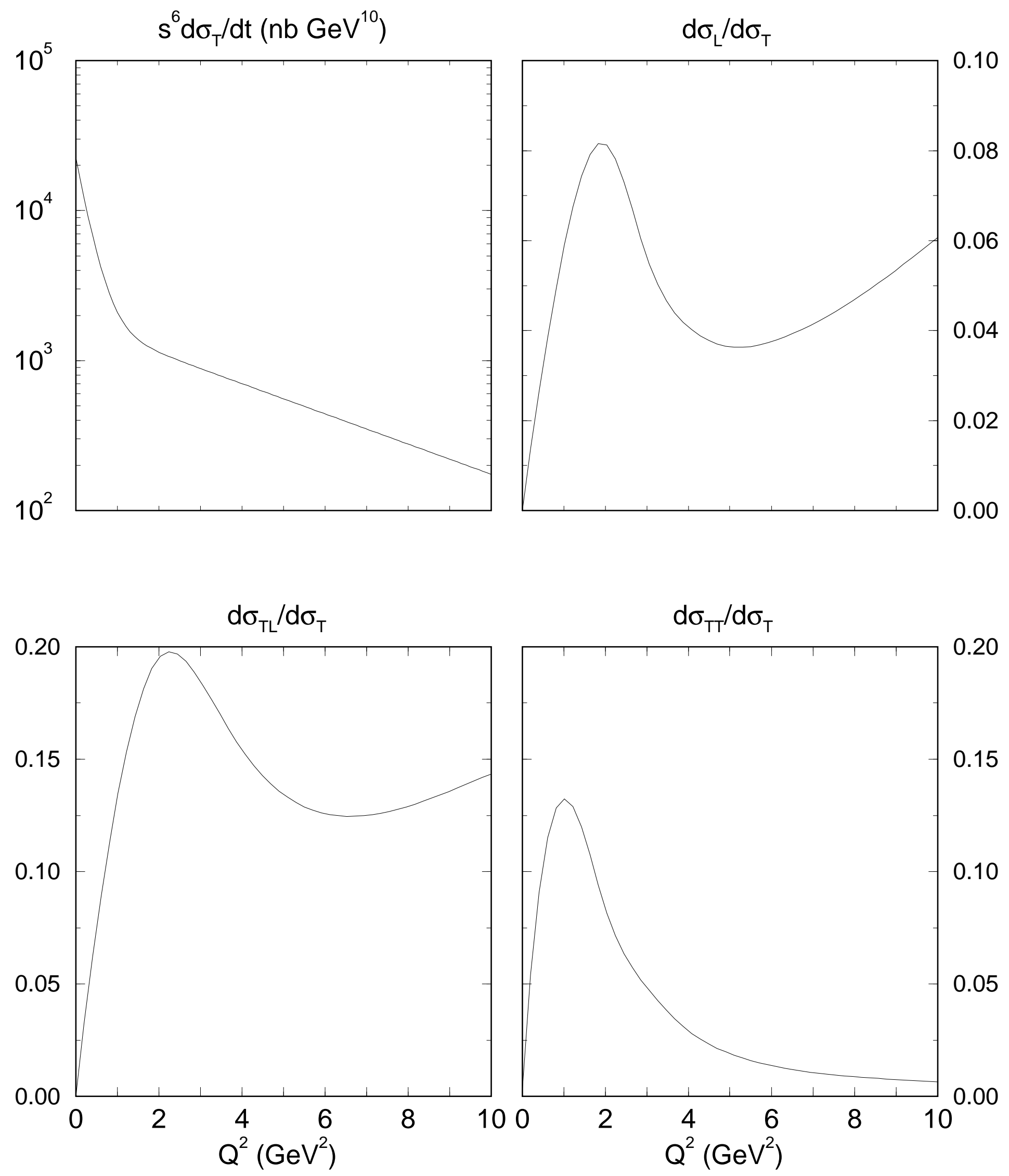

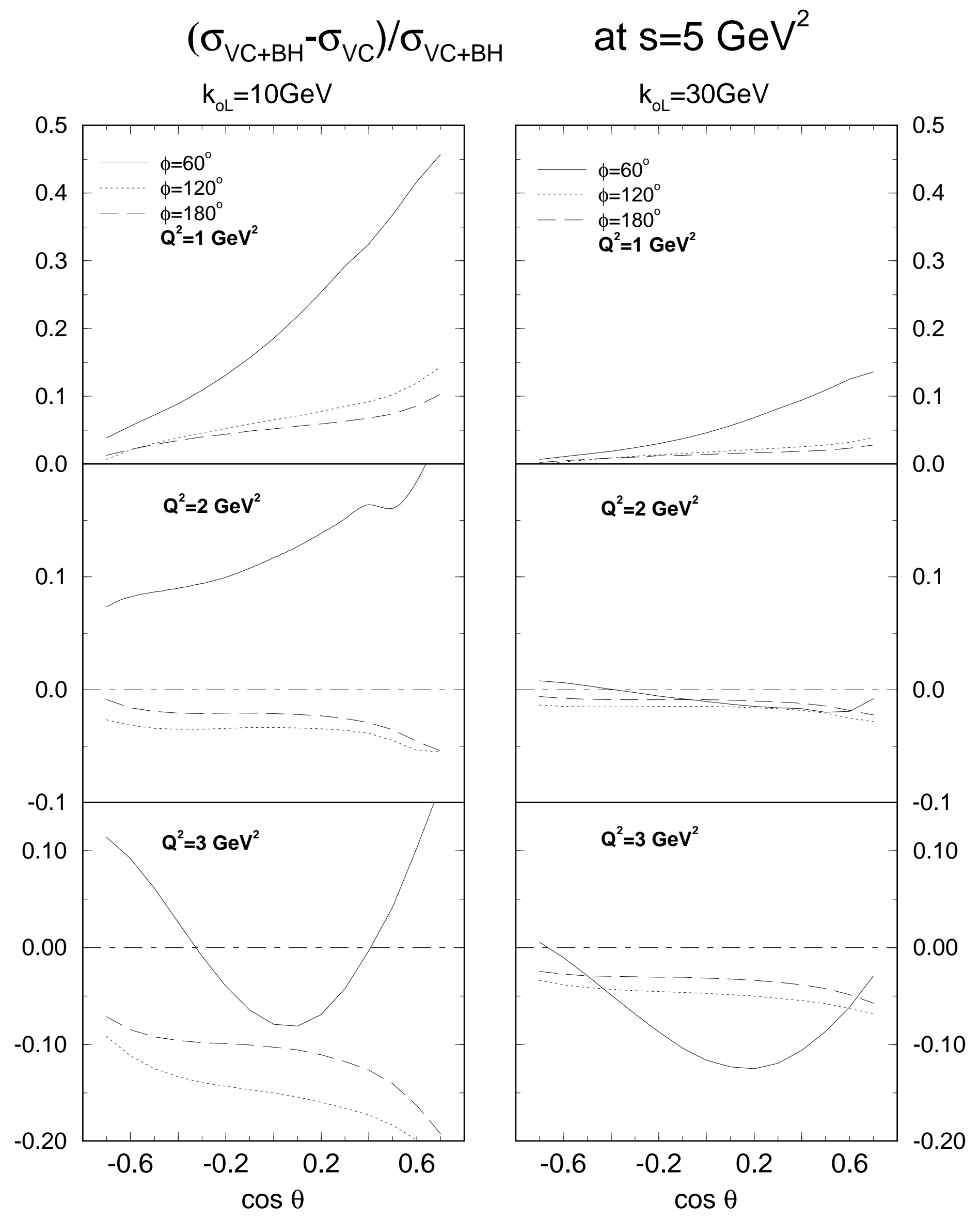

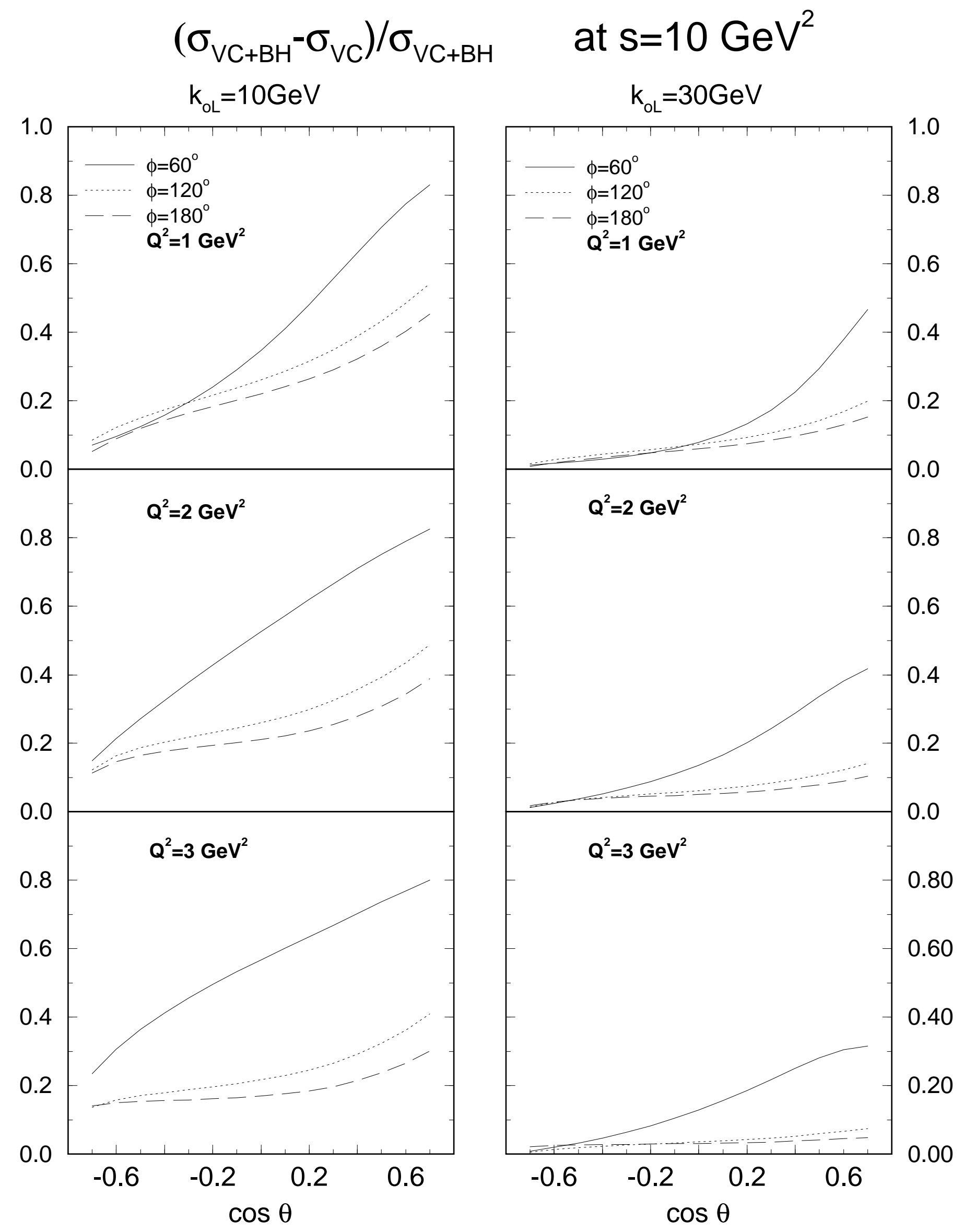

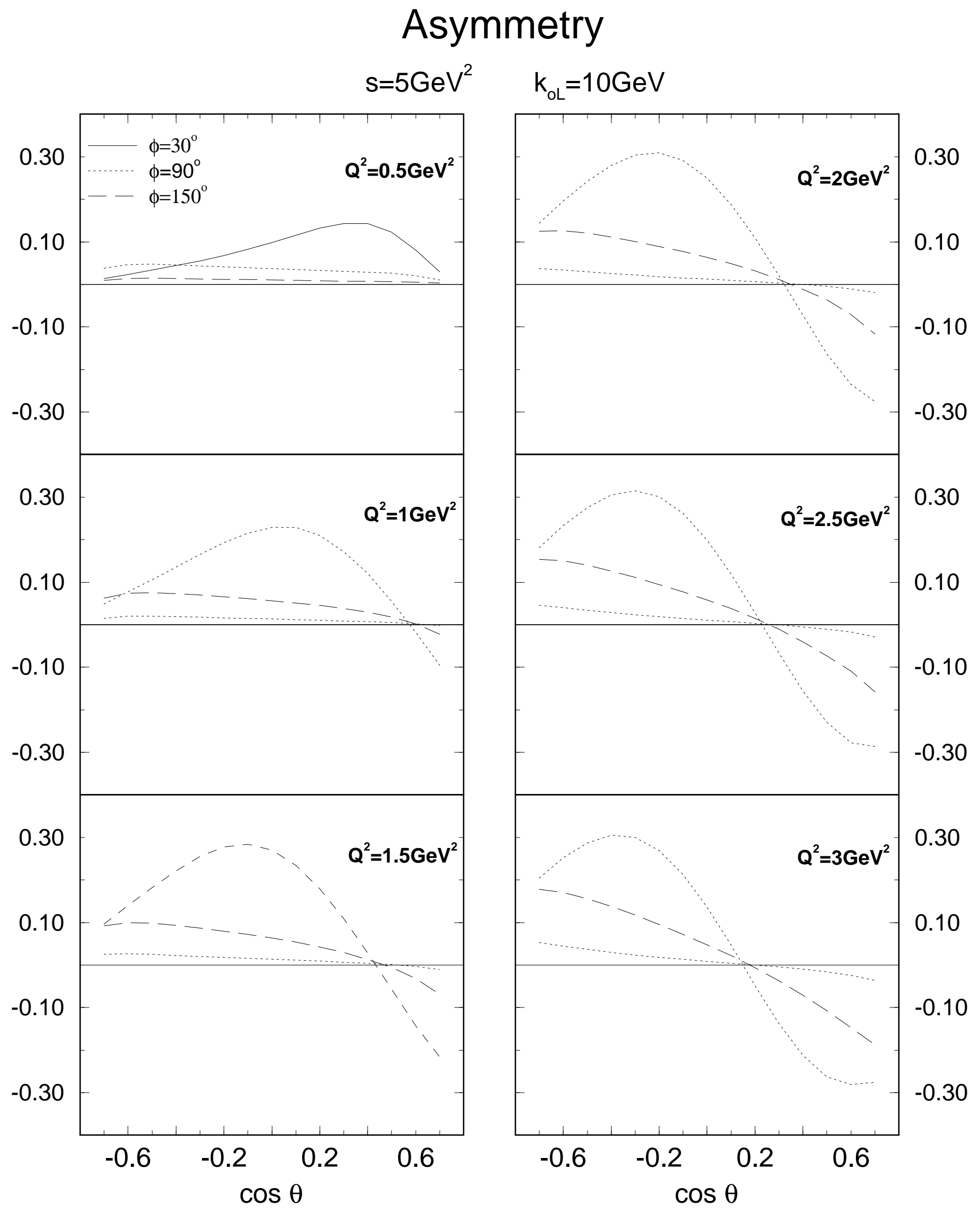


\section{Asymmetry prediction for CEBAF $\mathrm{s}=5 \mathrm{GeV}^{2}, \mathrm{Q}^{2}=1 \mathrm{GeV}^{2}, \mathrm{k}_{\mathrm{oL}}=6 \mathrm{GeV}$}
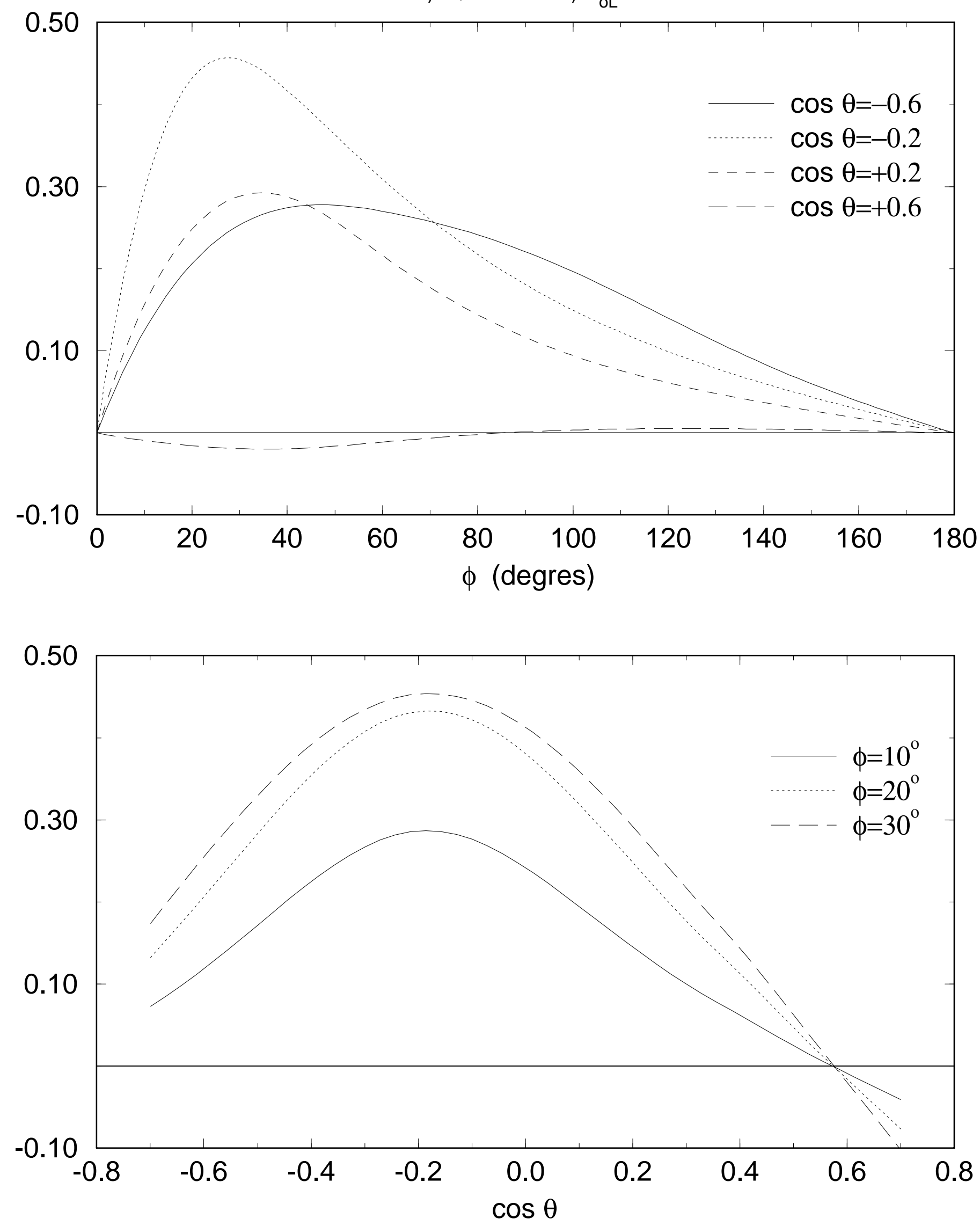